\title{
The Neuroscience of Dance: A Systematic Review of the Present State of Research and Suggestions for Future Work
}

Olivia Foster Vander Elst ${ }^{1}$, Nicholas H. D. Foster ${ }^{2}$, Peter Vuust ${ }^{1}$ \& Morten L. Kringelbach ${ }^{1,3,4}$

\author{
Addresses \\ ${ }^{1}$ Center for Music in the Brain, Department of Clinical Medicine, Aarhus University, Denmark \\ ${ }^{2}$ Formerly School of Law, SOAS University of London (Retired) \\ ${ }^{3}$ Centre for Eudaimonia and Human Flourishing, University of Oxford, UK \\ ${ }^{4}$ Department of Psychiatry, University of Oxford, UK
}

Table of Contents

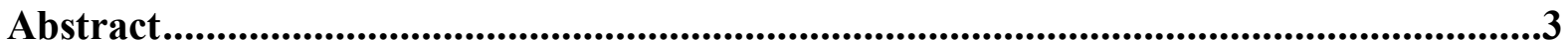

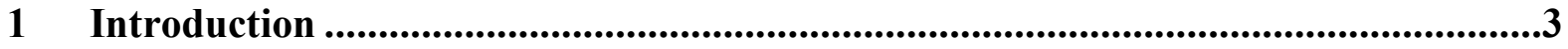

2 Difficulties associated with researching the neuroscience of dance ...........................6

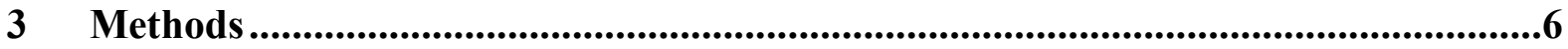

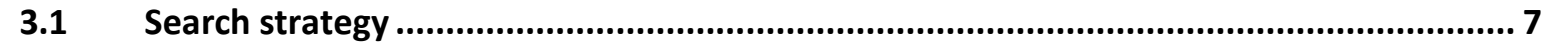

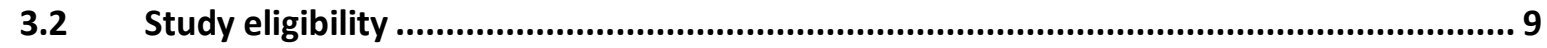

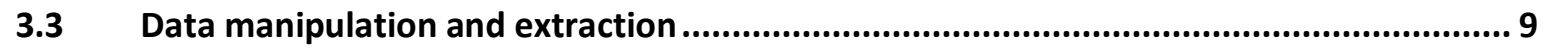

4 Review of individual studies by research area ..............................................10

4.1 Graphical and tabular representation of the number of studies published ................... 11

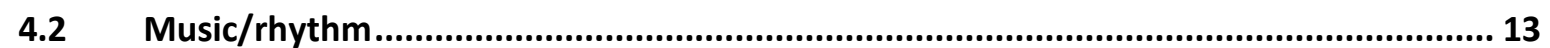

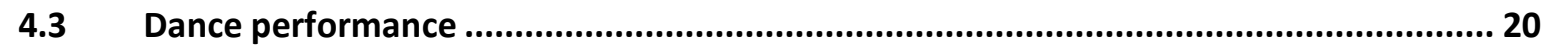

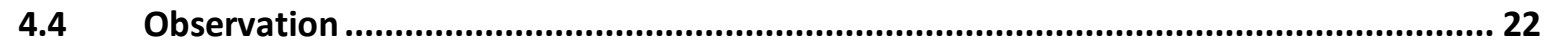

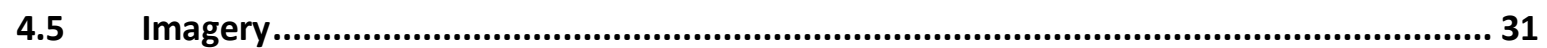

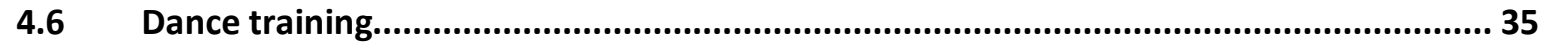

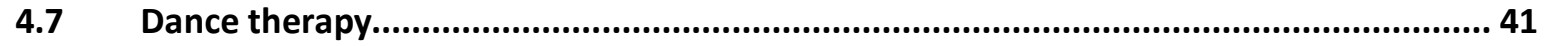

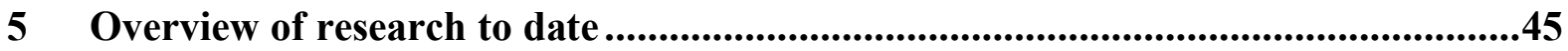

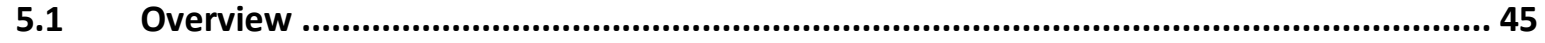




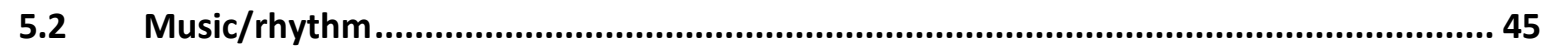

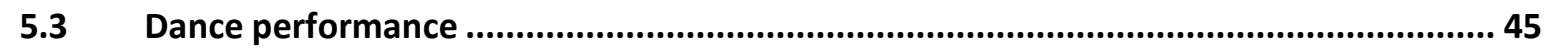

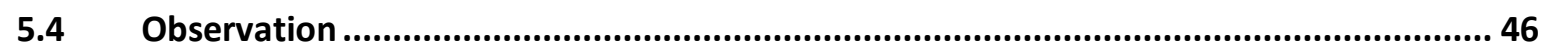

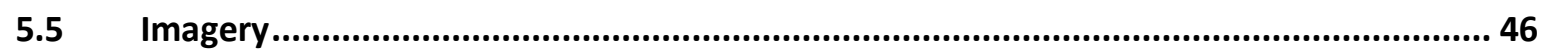

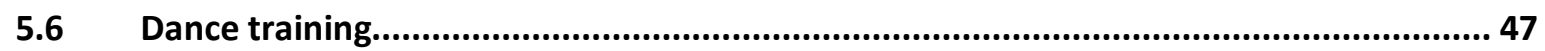

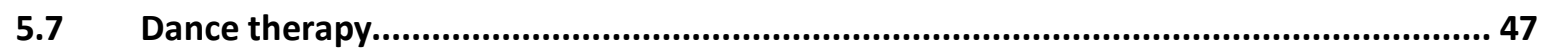

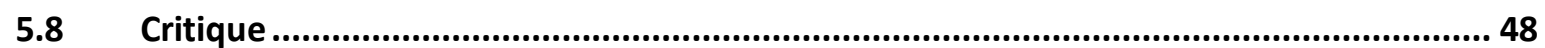

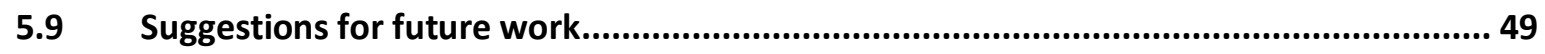

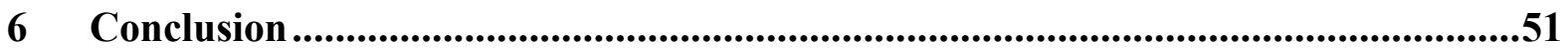

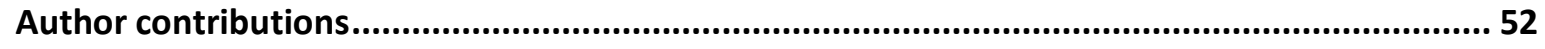

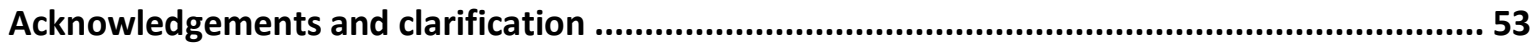

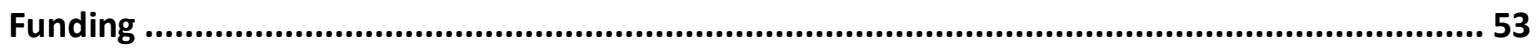

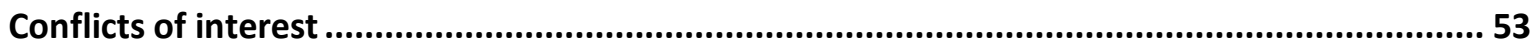

Appendix: Numerical overview of the studies reviewed................................................54

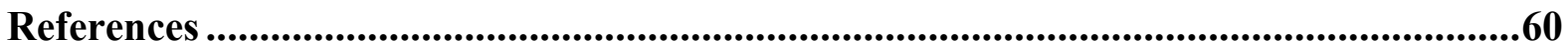




\begin{abstract}
Ancient and culturally universal, dance is important in many areas of life and brings pleasure, motivational, and health benefits. This is the first comprehensive review of research into its neuroscientific aspects. To produce it, we devised a map of the field, identified relevant papers using the PRISMA guidelines, then summarised and evaluated the results of those papers. We also suggest avenues for future research in: the interactive and collective aspects of dance; groove; dance performance; dance observation; and dance therapy. The interactive and collective aspects of dance constitute a vital part of the field, but have received almost no attention to date. More research has been conducted in the other areas, but they would benefit from additional attention.
\end{abstract}

Keywords: Dance; neuroscience of dance; collective and social aspects of dance; systematic literature review; eudaimonia; hedonia; motivation; pleasure; groove; dance performance; dance observation; dance imagery; dance training; music training; dance therapy; prediction; predictive coding model; improvisation; communication; interpersonal synchronisation; social entrainment; music.

\title{
1 Introduction
}

Dance can be easy to recognise, but defining it is more difficult. According to one definition, dance is "composed, from the dancer's perspective, of purposeful, intentionally rhythmical, and culturally patterned sequences of non-verbal body movements which are not ordinary motor activities, the motion having inherent and aesthetic value" (Hanna, 1987).

Ancient (Hanna, 1987; Karpati et al., 2015; Mithen, 2005) and culturally universal (Adler, 2009; Brown, 1991; Conard et al., 2009; Grafton and Cross, 2008; Levitin et al., 2018; Lomax, 1978; Spencer, 1985), dance has historically been important in many areas of life, serving valuable extrinsic goals such as social cohesion, ritual, and storytelling (Launay et al., 2016; Lewis, 2013; Lexová, 2012, p. 25; Merker et al., 2009; Merriam-Webster.com, 2021; Tarr et al., 2016, 2015). Dance is also frequently practised because it brings pleasure, including collective positive emotion, motivation, and health benefits. Dance can therefore contribute significantly to one's eudaimonia, the good life, discussed by Aristotle and many subsequent thinkers (Haybron, 2020).

The pleasurable and motivational aspects of dance seem to arise from the extended pleasure cycle which dance elicits. Pleasure cycles are characterised by distinct phases of 
wanting, liking, and satiety (Berridge and Kringelbach, 2015; Kringelbach and Berridge, 2009). In dance, the liking phase is extended compared to most sources of pleasure, producing longer overall pleasure cycles, with sustained periods of positive emotion (Foster Vander Elst et al., 2021; Kringelbach and Berridge, 2017; Stark et al., 2018). This extension of the pleasure cycle may be what induces people to, quite literally, dance the night away.

As a physical activity, neural processes are essential in dance. In order to understand dance thoroughly one must, therefore, study its neuroscientific aspects ("the neuroscience of dance"). In this article, we perform the first comprehensive review of this field. Karpati and colleagues published a review in 2015 (Karpati et al., 2015), but it was not intended to be comprehensive. Basso and colleagues' 2021 article (Basso et al., 2021) also contains useful material, but this is a "Hypothesis and Theory" article, and was not intended to provide a review of the neuroscience of dance.

To produce the review, we devised a map of the field, identified relevant papers using the PRISMA guidelines, then summarised and evaluated the results of those papers. As research varied greatly over time and by area (for example, dance training is investigated in 54 papers, but dance therapy is only investigated in 15 papers), we have produced a tabulated chronology. We also provide a bibliography of papers containing original, neuroscientific, results in each area in the supplementary information.

When listening to music, the brain constantly generates predictions based on an underlying model, guiding our perception and actions, as outlined in the Predictive Coding of Music model. In this model, it is proposed that music processing, including perception, action, emotion, and learning, is an integrative, recursive Bayesian process, by which the brain attempts to minimize the prediction error between lower-level sensory input and the brain's top-down predictions (Heggli et al., 2019; Vuust et al., 2009; Vuust and Frith, 2008).

Actions carried out in response to music may include dancing. Prediction is also important in dance, as dancers must predict what will happen next in the music, the movement of their dance partner, and the movement of other dancers on the dance floor. Furthermore, in a partner dance, the leader directs the dance, planning it spontaneously in an improvisatory fashion. The leader must decide what moves to perform, and communicate physical cues to the follower such that the follower is able to respond quickly and easily. The follower must predict what moves the leader is going to do, but must be ready to respond and adapt immediately if these predictions turn out to be incorrect. Given the important role of prediction in dance, and the fact that the PCM model may be extended to explain the brain 
mechanisms of collective music-making (Vuust et al., 2021), this model may also be helpful in explaining the brain mechanisms of the social and interactional aspects of dance.

Dance is therefore a powerful tool for studying: (1) prediction in the brain; (2) the underlying brain mechanisms of social interaction, including communication, social entrainment, and interpersonal synchronization; (3) improvisation, using unchoreographed dance; (4) the integration of movement and cognition; (5) movement synchronisation, both to music and to other people; (6) sensorimotor integration; (7) the effects of long-term, intensive, practice of sensorimotor skills (Karpati et al., 2015); (8) action-perception coupling (Bläsing et al., 2012); (9) advanced motor control; (10) brain plasticity (Karpati et al., 2015); (11) brain-behaviour relationships and their development (Karpati et al., 2015); and (12) neuroaesthetics.

Research in the field has yielded important results relating to neural activity and changes in brain structure relating to dance. However, much work remains to be done. Most research to date has been focused on individuals, and we therefore suggest that a potentially fruitful subject of future research is the collective experience of dance, especially those of its aspects which relate to communication, social entrainment, and interpersonal synchronization. Since dance is a highly interactive and collective activity, such research could also be a powerful tool for studying the predictive and social interaction mechanisms of the brain.

Other areas that could benefit from further research are groove, dance performance, dance observation (the active observation of dance performance), and dance therapy. To date, research into groove has not been concerned with dance, despite the close association of the two phenomena, and we therefore suggest that more research be conducted on the neuroscience of groove directly involving dance. As for dance performance, recent advances in technology may alleviate the difficulties of investigating it directly. Indeed, two studies exploiting these advances are presently in preparation at the Universities of Groningen and Houston. More use of live, in-person, observation of dance would be beneficial for research into dance observation. Research on the neuroscience of dance therapy could be furthered and improved by carrying out larger scale studies, investigating a wider range of health conditions and, ideally, including both active and "no-change" control groups, and using comparable dance interventions. 


\section{Difficulties associated with researching the neuroscience of dance}

Numerous difficulties are associated with researching the neuroscience of dance. Some of these difficulties arise in experimental design and ecological validity, because elements such as stage changes and lighting cannot be recreated in a laboratory (Dale et al., 2007).

The principal challenge, however, is technological, as dance requires the person being scanned to move. Electroencephalography (EEG) headsets and function Near-Infrared Spectroscopy (fNIRS) allow for investigation of the neural activity of moving people, but have low spatial resolution in the brain. Techniques with higher spatial resolution, such as functional Magnetic Resonance Imaging (fMRI) and magnetoencephalography (MEG), do not allow for movement. Recent advances in techniques such as Mobile Brain/body Imaging (described in (Barnstaple et al., 2020)) may change this in the near future, but no studies have been published to date in which this technique has been used to study dance performance. We are, however, aware of at least two studies in preparation by Marieke Van Vugt's group at the University of Groningen and José Contreras-Vidal's group at the University of Houston, exploiting such advances in technology to research dance performance directly.

To work around these difficulties, some research has been carried out on people performing activities which are similar to dancing, but are of such a nature that the participants can be scanned. The principal substitute activities are: lying supine in a scanner, with the feet resting on an inclined plane, and performing tango steps; and performing a dance video game based on Dance Dance Revolution ${ }^{\mathrm{TM}}$.

Many tapping/joint flexion studies have also been carried out, in which neural activity associated with small movements to music has been investigated. However, we consider this activity too far removed from dancing to have sufficient relevance to the neuroscience of dance unless other aspects of the study are relevant to dance, e.g. investigating the effects of dance training on neural activity during such activities.

Other attempts to circumvent these difficulties have led to a wealth of research into activities related to dance, such as dance observation and imagery. Research in these areas contributes to our knowledge of the neuroscience of dance performance, as well as yielding results that are valuable in their own right.

\section{Methods}

As the neuroscience of dance is a relatively new field, it had not previously been mapped. This was, therefore, our first task. By "mapping the field" we mean: (1) categorising the 
research areas, or parts of research areas, relevant to the neuroscience of dance; (2) deciding which of these to include or exclude; (3) setting out the reasons for those choices; and (4) ascertaining the relationships of the research areas to each other. In this process, we evaluated a broad range of research areas in terms of their proximity to dance performance, and their utility for research into its neuroscientific aspects.

The research areas which we decided to include are:

1. the phenomena which are usually required for dance to occur and continue (“music/rhythm");

2. dance performance;

3. activities which are closely related to dance performance and which can therefore also act as research proxies for it, as well as yielding valuable results in their own right:

a. the active observation of dance performance ("observation"); and

b. motor imagery relevant to dance ("imagery");

4. dance training, an activity which, in neurological terms, is very close to, and merges with, dance performance; and

5. dance therapy, the major contemporary application of dance, and the area most likely to benefit practically from further neuroscientific investigation.

The terms in inverted commas are defined more fully in sections 4.2, 4.4 and 4.5 respectively. In some of the areas mentioned, only some of the research is relevant to the neuroscience of dance, so we have only included such research. We provide an outline of each research area and explain the reasons for including it, or part of it, in section 4.

\subsection{Search strategy}

We conducted a major literature search in December 2019, which was augmented at regular intervals up to and including February 2021. The main resources used were the NCBI database, PubMed, and Google Scholar. Typical general search terms were "neuroscience of dance," "neuroscience," and "dance." For specific research areas, typical search terms were "groove," "auditory processing," "dance observation," “dance/motor imagery," "dance imagery," "dance training," "dance therapy," and "dance/movement therapy." We did not impose conditions on the language of the article nor, given the short track record of the field, did we impose conditions on publication year. These searches resulted in 4350 articles.

We searched through conference proceedings and traced references backward and forwards in the articles we had already identified as potential candidates for inclusion. We 
also read the publication lists of the authors of these papers. In these searches we identified a further 27 articles.

In the final stage of the process we contacted several researchers in the field and asked them for recommendations. No further articles were identified from this process.

We therefore identified 4377 articles in total. The removal of duplicates reduced this number to 4005 . The titles and abstracts of these articles were screened for mentions of dance or related terms, reducing the number to 364 full text articles. Of these, 93 fulfilled the criteria set out in section 3.2 and were therefore included in the review. The number of articles at each stage of this process is summarised in figure 1.
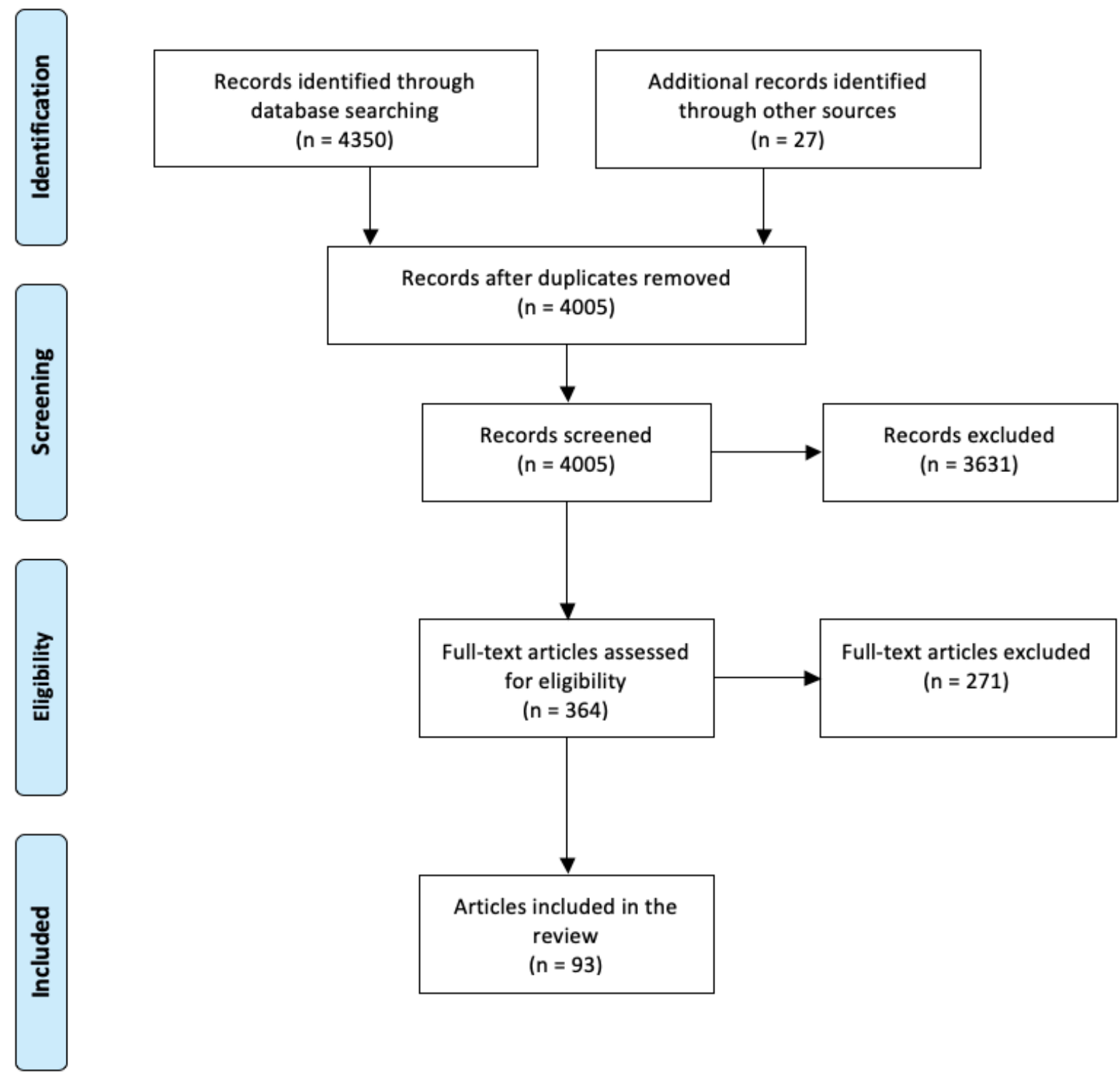

Figure 1: PRISMA flowchart showing the number of articles at each stage of the literature search. Adapted from (Moher et al., 2009). 


\subsection{Study eligibility}

The criteria used in the selection of the articles we included were:

- the nature of the subject matter: only studies in the included research areas, or relevant parts of an included research area;

- the nature of the studies: only original studies, not, e.g., review articles with no original, experimental, data; and

- the nature of the results: only studies containing neuroscientific results, not, e.g., studies containing solely psychological or questionnaire-based results.

In order to assess the eligibility of the 364 full text articles, we searched all titles and abstracts for terms related to dance. For the remaining 241 articles, we read the abstracts in detail and used these to determine if the article should be included. Based on the criteria above, we included 93 studies.

\subsection{Data manipulation and extraction}

We created a database of information on the 241 full text articles. This enabled us to include and manipulate data in addition to the standard referencing information. The additional data for each article were: (1) a numerical ID; (2) the abstract; (3) the research area or areas for which it was relevant; (4) neuroimaging techniques employed; (5) the regions of the brain mentioned in it; (6) keywords (those provided, and additional ones chosen by us); and (7) whether or not it was included in the review.

\subsubsection{Selection and categorisation of articles, and extraction of results}

In order to select which articles to include, we: (1) printed all of the abstracts with their numerical IDs; (2) read the abstracts; (3) discarded those which did not meet the criteria outlined in section 3.2; and (4) categorised the remaining articles by research area, using multiple hard copies of the abstract for articles concerned with more than one research area.

Within each research area, we used the hard copies of the abstracts to categorise the results set out in the article, e.g. for an article concerned with dance observation, we further categorised the results as relating to aesthetic judgements, dance training etc.. Finally, we: (1) read each article in full; (2) identified and recorded in the database its neuroscientific results 
and the research area or areas to which those results related; (2) identified and recorded the broader relevance, if any, of those results; and (2) used this data to draft section 4.

\subsubsection{Extracting numerical information about the articles reviewed}

We used the database to extract numerical information about the articles reviewed. One aim of this process was to assign a numerical and objective, rather than approximate and judgment-based, value to the quantity of studies published. To our knowledge, the only other review article in a related field in which this technique has been used is Cross et al. (Cross et al., 2014). Our method was devised independently.

Another aim of this process was to evaluate the progression of the field over time, using the number of studies published each year. This is of course approximate, as it does not take into account the limitations of evaluating a field solely on the number and date of studies published. These include the discrepancy in time between research being undertaken and studies published, the potential disparity between the number of studies published and the number of research projects undertaken, and various other measures of the amount and importance of research activity. However, it does help to give a general idea of the development of the field.

An overview of this information is given in section 4.1. More detailed information is set out in the Appendix.

\section{Review of individual studies by research area}

This section contains a review of the 93 individual studies we identified as relevant to the field of the neuroscience of dance. The review is organised by research area, with each research area. The research areas are: (1) music/rhythm; (2) dance performance; (3) observation; (4) imagery; (5) dance training; and (6) dance therapy.

In section 4.1, we provide graphical and tabular representations of the number of studies published. Each of the subsequent sub-sections consists of: (1) an outline of the research area; (2) the reasons for its inclusion, or partial inclusion, in the field; (3) a summary of numerical publication information; and (4) summaries of the key results of the studies relating to that area. Some studies relate to more than one area, so appear in more than one sub-section.

In order to avoid undue repetition of phrases such as "it was found that" and "in [a/another/further] study:" (1) study results are presented as facts without preliminary wording indicating that they are the results presented by the researchers - this is a purely 
stylistic matter, and no opinion regarding the status of the results presented should be implied from it; and (2) where appropriate, in-text citations are used as abbreviations for study titles.

Frequency bands of brain activity are italicised for ease of reading (alpha, beta, gamma, and theta).

\subsection{Graphical and tabular representation of the number of studies published}

The total number of studies published in each area and each year, as well as the total number of unique studies published in each year, are shown in figure 2. These data are also set out in table 1. Individual graphs of the number of studies published in each area, in each year, are set out in the Appendix.

We do not include research on beat perception in the numerical data. Although this research is relevant to the neuroscience of dance, and we include a brief overview of a selection of that research, it is not sufficiently relevant to merit detailed textual or numerical consideration.

As noted in section 3.1, we found 93 articles in which original research was presented on the neuroscience of dance. Research in the field started in earnest in 2005, and out of 92 articles published since 2005, 61 were published in 2014 or later. 
(a)

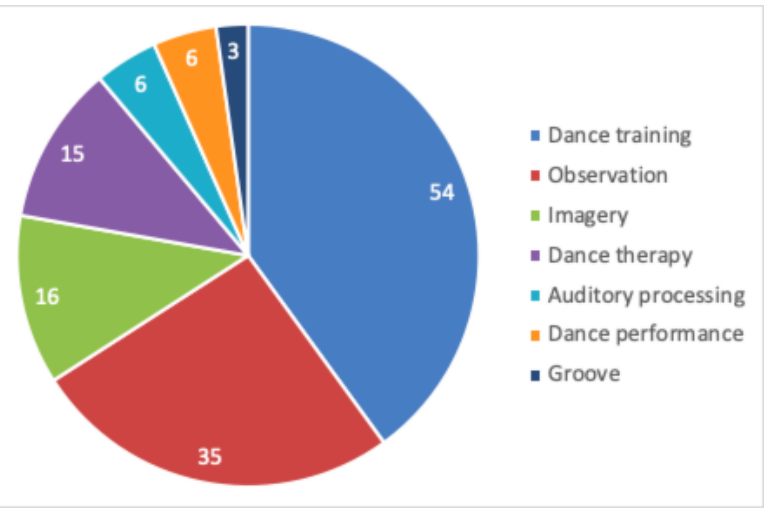

(b)

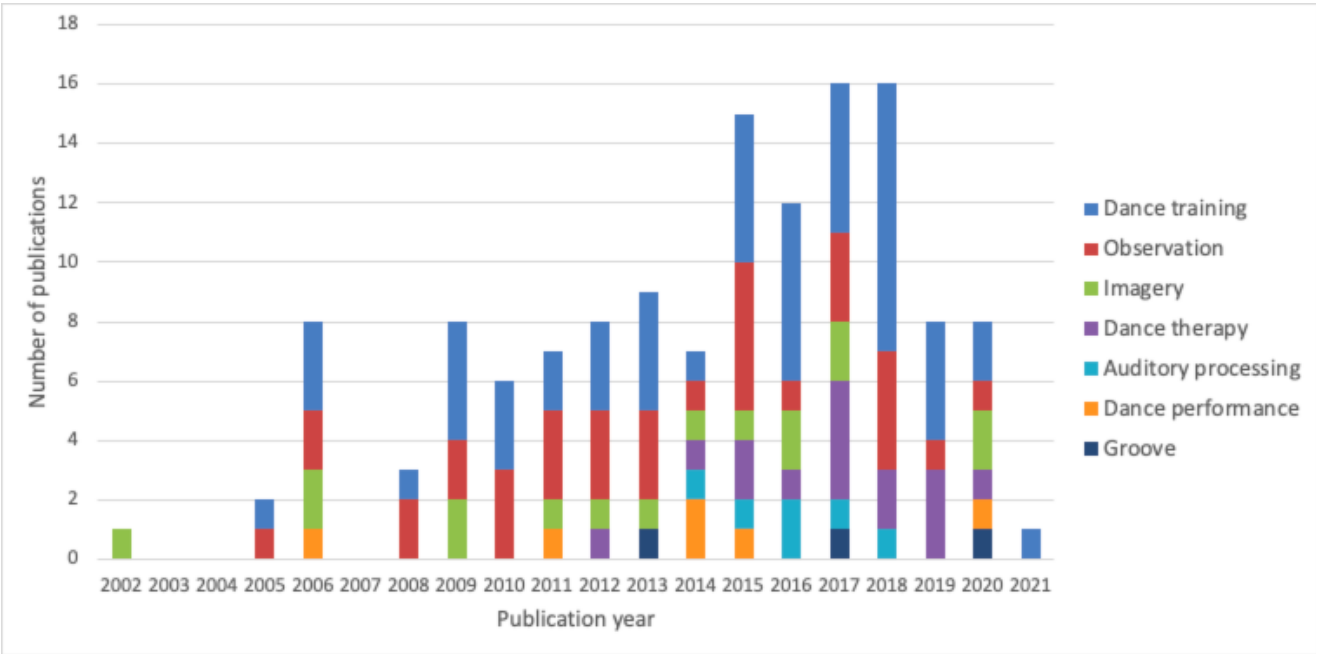

(c)

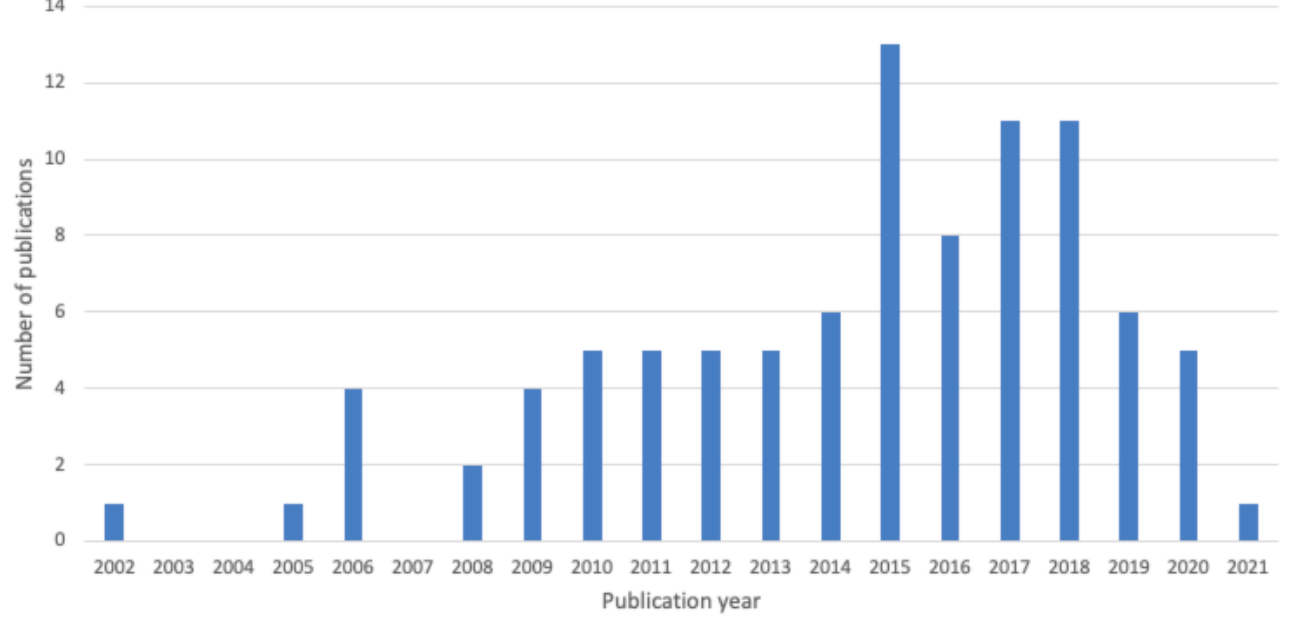

Figure 2: (a) Total number of studies published concerned with each area, listed in clockwise order of decreasing number of studies. (b) Number of studies published each year concerned with each area, listed in order of decreasing number of studies. Some studies are included in more than one area. (c) Total number of individual studies published each year. 
Table 1: All studies published in each area, arranged by year, listed in order of decreasing number of studies, and total number of unique studies published each year.

\begin{tabular}{|c|c|c|c|c|c|c|c|c|}
\hline & \multicolumn{8}{|c|}{ Number of studies } \\
\hline $\begin{array}{c}\text { Publication } \\
\text { year }\end{array}$ & $\begin{array}{c}\text { Dance } \\
\text { training }\end{array}$ & Observation & Imagery & $\begin{array}{l}\text { Dance } \\
\text { therapy }\end{array}$ & $\begin{array}{l}\text { Auditory } \\
\text { processing }\end{array}$ & $\begin{array}{c}\text { Dance } \\
\text { performance }\end{array}$ & Groove & $\begin{array}{c}\text { Total } \\
\text { unique } \\
\text { studies }\end{array}$ \\
\hline 2002 & & & 1 & & & & & $\mathbf{1}$ \\
\hline 2003 & & & & & & & & \\
\hline 2004 & & & & & & & & \\
\hline 2005 & 1 & 1 & & & & & & 1 \\
\hline 2006 & 3 & 2 & 2 & & & 1 & & 4 \\
\hline 2007 & & & & & & & & \\
\hline 2008 & 1 & 2 & & & & & & 2 \\
\hline 2009 & 4 & 2 & 2 & & & & & 4 \\
\hline 2010 & 3 & 3 & & & & & & 5 \\
\hline 2011 & 2 & 3 & 1 & & & 1 & & 5 \\
\hline 2012 & 3 & 3 & 1 & 1 & & & & 5 \\
\hline 2013 & 4 & 3 & 1 & & & & 1 & 5 \\
\hline 2014 & 1 & 1 & 1 & 1 & 1 & 2 & & 6 \\
\hline 2015 & 5 & 5 & 1 & 2 & 1 & 1 & & 13 \\
\hline 2016 & 6 & 1 & 2 & 1 & 2 & & & 8 \\
\hline 2017 & 5 & 3 & 2 & 4 & 1 & & 1 & 11 \\
\hline 2018 & 9 & 4 & & 2 & 1 & & & 11 \\
\hline 2019 & 4 & 1 & & 3 & & & & 6 \\
\hline 2020 & 2 & 1 & 2 & 1 & & 1 & 1 & 5 \\
\hline 2021 & 1 & & & & & & & 1 \\
\hline Total & 54 & 35 & 16 & 15 & 6 & 6 & 3 & 93 \\
\hline
\end{tabular}

\subsection{Music/rhythm}

For the purposes of the neuroscience of dance, we group into one category studies on the phenomena which bring dance about and ensure its continuance. Since music is so important in dance, rhythm is by far the most important component of music in this context (Leisman 
and Aviv, 2020), and music and rhythm are the linking factors in these phenomena, we denominate this category "music/rhythm." We divide this category into: (1) auditory processing; and (2) groove; and sub-divide auditory processing into: (1) music; (2) rhythm; (3) non-musical sounds; and (4) beat perception.

Many studies have been undertaken on the neuroscience of these phenomena, but most of them are not relevant to the neuroscience of dance. In this article, therefore, we only review those studies that are directly related to the field.

\subsubsection{Auditory processing}

Dance is almost always performed to music and rhythm is the most important element of music in dance (Leisman and Aviv, 2020). Indeed, dance is sometimes performed to rhythm alone, in a form not necessarily classified as music. Furthermore, some non-musical sounds, such as the sound of dancers' breath or the sound of their feet hitting the ground, are also relevant.

The auditory processing of these phenomena is a key part of the neural activity which occurs both before and during dance. We therefore include in this review research relating to the auditory processing of these phenomena when such auditory processing is directly relevant to the neuroscience of dance.

The importance of rhythm to dance means that the ability to perceive temporal regularity in musical rhythms (beat perception) (Bouwer et al., 2016), is also relevant to the neuroscience of dance. However, as noted in section 4.1, although research on beat perception is of some significance, it is not sufficiently relevant to merit detailed consideration. We therefore include, in section 4.2.1.2, only a brief overview of a selection of that research.

\subsubsection{Auditory processing directly relevant to the neuroscience of dance}

The subjects of these studies in this area involved: (1) imagining a metre while participants listened to identical, evenly spaced, tones; (2) moving to a metre; and (3) the effect of dance and musical training on auditory processing.

\section{Imagining a metre}

A metre is a rhythm in which there are regular, recurring patterns of strong and weak beats (Vuust et al., 2006). This pattern may be present in the music/rhythm itself, or may be purely imagined, and is especially important in predicting future events in the music/rhythm (Vuust and Witek, 2014). 
Metre is of central importance in dance. Common metres are made up of three or four beats. In a metre of three beats, used in dances such as the waltz, every third beat is emphasised (STRONG weak weak STRONG weak weak). In many other dance styles, such as salsa, every fourth beat is emphasised (STRONG weak weak weak STRONG weak weak weak). Paso Doble uses a metre of two beats, in which every second beat is emphasised (STRONG weak STRONG weak). Marching, which is not usually classified as dance but resembles it in some ways, is also performed to a metre of two beats.

In (Fujioka et al., 2015), the effects of imagining a metre were investigated while participants listened to identical, evenly spaced, tones. Brain wave activity in the region of 13-30 Hz, the beta band, was investigated. A decrease in beta band power, related to the beat, occurred $200 \mathrm{~ms}$ after it, and was followed by an increase in power leading up to the onset of the next beat. This increase was probably predictive. When the participants imagined the metre of either a march or a waltz while listening to these tones, a greater increase in beta band power occurred on the strong beat than on the subsequent weak beat. The regions of the brain involved were: (1) the auditory and sensorimotor cortices; (2) the frontal, parietal, and inferior temporal lobes; and (3) the cerebellum. This activity may reflect "a translation of timing information to auditory-motor coordination" (Fujioka et al., 2015).

\section{Moving to a metre}

Two other studies (Chemin et al., 2014), (Morillon and Baillet, 2017) involved listening to identical tones and movement. Like the imagination of a metre, movement affects auditory processing. The effect is felt both when one moves and when one observes someone else moving.

In (Chemin et al., 2014), participants listened to a repeated rhythmical sequence of identical tones. They then moved to the rhythm in a specific metre, and after having moved to the metre, listened to the rhythm again. Their neural responses to rhythms at frequencies related to that metre were significantly increased after moving to it.

In (Morillon and Baillet, 2017), conscious rhythmic movement improved temporal predictions relevant to rhythms which the participants listened to. It appears that these predictions were encoded by the left sensorimotor cortex through interdependent beta and delta neural oscillations originating from this region, which were directed towards auditory regions of the brain. In addition, during overt rhythmic movement, there was greater rightlateralized activity in frontoparietal associative regions, and this was related to temporal predictions. 
In (Reason et al., 2016), a study on dance observation, there was greater inter-subject correlation in the presence of music in a left anterior region of the superior temporal gyrus (STG) involved in complex audio processing. In the absence of music, there was greater inter-subject correlation in the parietal and occipitotemporal cortices, and in a posterior region of the STG.

It seems from these results that, when we listen to identical, evenly spaced, tones, motor regions of the brain play key roles in providing temporal and contextual information to sensory regions of the brain (Morillon and Baillet, 2017). It also appears that movement to a metre helps to internalise it through this neural activity, improving temporal predictions relevant to the metre (Chemin et al., 2014). This is crucial in dance, where the dancers must synchronise their movements to the music. The difference in activation of the STG when dance was observed with and without music is believed to be due to sounds made by the dancer being observed, such as the breath, providing "a more salient auditory signal than music in primary auditory regions" of the brain (Reason et al., 2016).

\section{Dance training and musical training}

As might be expected from the results of the above studies (Chemin et al., 2014; Morillon and Baillet, 2017; Reason et al., 2016), dance training affects auditory processing, as does musical training. These effects were investigated in two studies (Poikonen et al., 2016, 2018a) in which the neural activity of three groups was investigated as they listened to an excerpt of Shchedrin's Carmen Suite, a ballet suite arrangement of Bizet's opera. The three groups were: (1) dancers; (2) musicians; and (3) a control group who did not have any significant dance or musical training ("non-musicians/dancers").

In (Poikonen et al., 2016), event-related potentials (changes in neural activity related to specific musical events), were investigated, especially the P50, N100, and P200 responses. In these labels, "P" and "N" represent positive and negative changes in potential respectively; the number refers to the approximate number of milliseconds after the event.

When the timbre of the music changed, the P50 was greater in dancers than in either musicians or non-musicians/dancers, suggesting that the dancers had better pre-processing than either of the other groups. The N100 response was larger in non-musicians/dancers than in either dancers or musicians, which might have been a counter-effect of the greater P50 observed in dancers.

In (Poikonen et al., 2018a), the synchrony of different brain wave frequency bands (theta 4-8 Hz, alpha 8-13 Hz, beta $13-30 \mathrm{~Hz}$, and gamma 30-48 Hz) across different electrode channels on the participants' scalp was investigated. When participants observed dance in the 
presence of music, the dancers had greater synchrony across different electrodes in the theta and gamma bands than when they observed silent dance. When the musicians observed dance in the presence of music, they had less synchrony across different electrodes in the alpha and beta bands than when they observed silent dance.

It appears from these results that dancers have better pre-processing of changes in timbre in music than either musicians or non-musicians/dancers (Poikonen et al., 2016). It also appears that different neural processes occur when dancers, musicians, and nonmusicians/dancers listen to music, and expertise in both dance training and music training "seem[s] to shape the perception of both music and dance" (Poikonen et al., 2018a). The unconscious awareness of changes in timbre which this gives dancers may help them to adapt their movements to reflect the character of the music more successfully when such changes occur.

\subsubsection{Beat perception}

In several studies, activity occurred in neural networks associated with both motor timing and auditory-motor integration during beat perception (Araneda et al., 2017; Bengtsson et al., 2009; Burunat et al., 2017; Chapin et al., 2010; Chen et al., 2008; Grahn and Brett, 2007; Grahn and Rowe, 2013, 2009; Kung et al., 2013; Schubotz et al., 2000; Thaut et al., 2014). Furthermore, the basal ganglia appear to perform a crucial role in beat perception (Matell and Meck, 2004; Merchant et al., 2015; Teki et al., 2011), and are part of corticostriatal "motor circuits" which may support both motor functions and motivational functions relevant to the pleasurable desire to move to music (Alexander et al., 1986; Haber, 2003; Matthews et al., 2020). It also appears that the putamen and the supplementary motor area, both part of the corticostriatal circuits, are involved in beat and metre perception (Araneda et al., 2017; Q. Li et al., 2019).

In response to a strong beat, neural activation has been observed in premotor, prefrontal, and parietal regions (Bengtsson et al., 2009; Danielsen et al., 2014; Grahn et al., 2011; Grahn and McAuley, 2009; Grahn and Rowe, 2009; Grahn and Schuit, 2012; Mcauley et al., 2012; Schubotz et al., 2000; Schubotz and von Cramon, 2001). Furthermore, the caudate formed circuits with prefrontal and parietal regions (Jarbo and Verstynen, 2015), and these circuits were implicated in rhythmic expectation (Trost et al., 2014).

It is clear from these results that several motor regions of the brain are engaged when listening to music, an engagement which is related to the formation of rhythmic expectations, and that movement preparation also occurs when listening to music. It has been suggested 
that this movement preparation is used to "test" the rhythmic expectations formed (Matthews et al., 2020).

\subsubsection{Groove}

Groove is the pleasurable sensation of wanting to move to music/rhythm (Janata et al., 2012; Senn et al., 2018; Madison, 2006). Acting on this desire can result in dance. Groove is a subjective sensation, but there is considerable consistency across random samples of people in assigning degrees of groove to excerpts of music, as ascertained by asking participants to assign various adjectives to musical excerpts (Madison, 2006; Stupacher et al., 2016). The results of these assignments are used in defining music/rhythm as high-groove or low-groove.

Groove is most strongly felt when music/rhythm has medium levels of syncopation. Syncopation is the appearance of a beat on a metrically weak accent, preceding a rest on a metrically strong accent (Longuet-Higgins and Lee, 1984)) (Matthews et al., 2019; Witek, et al., 2014). Too little syncopation is perceived as boring, too much is too complex, but a medium amount is felt to be interesting, and creates a desire to "fill in" the gaps left by the syncopated rhythms not sounding on metrically strong accents (Witek, 2017).

Groove is a key factor linking music and dance, so its neuroscientific aspects are directly relevant to the study of the neuroscience of dance. We therefore include studies on the neuroscience of groove in this review, even though dance was not specifically investigated in any of them.

The subjects of the studies in which the neuroscience of groove was investigated were: (1) a comparison of high-groove and low-groove music/rhythm; and (2) groove and musical training.

\subsubsection{Comparing high-groove and low-groove music/rhythm}

High-groove and low-groove music/rhythm affect the nervous system differently. In (Stupacher et al., 2013), listening to high-groove music modulated corticospinal excitability in both musicians and non-musicians more than listening to low-groove music or noise. In (Matthews et al., 2020), listening to high-groove rhythms resulted in greater activity in reward-related regions of the brain than listening to low-groove rhythms. The regions concerned included: (1) the nucleus accumbens; (2) the caudate; and (3) the medial orbitofrontal cortex. Greater activity was also recorded in regions of the brain associated with beat-based timing, including: (1) the putamen; (2) the supplementary motor area; and (3) the prefrontal and parietal cortices. 
It seems from these findings that the nervous system is more engaged when listening to high-groove music/rhythm than when listening to either low-groove music/rhythm or noise (Stupacher et al., 2013), and that the sensation of groove which results from listening to highgroove music/rhythm "is driven by a combination of motor and reward regions in the brain" (Matthews et al., 2020).

\subsubsection{Groove and musical training}

People who have undertaken musical training are generally more adept at perceiving rhythms, but this training has no significant effect on the degree of groove they perceive (Stupacher et al., 2013; Witek, et al., 2014). It does, however, affect their neural responses when listening to high-groove music/rhythm, low-groove music/rhythm, or noise (Matthews et al., 2020; Stupacher et al., 2017, 2013).

In (Stupacher et al., 2017), musicians had increased neural activity in motor-related areas of the brain when listening to high-groove music/rhythm, but not when they listened to lowgroove music/rhythm. This effect was not observed in non-musicians.

In (Stupacher et al., 2013): (1) musicians had higher corticospinal excitability than nonmusicians when listening to high-groove music; (2) there was no difference between musicians and non-musicians when they listened to low-groove music; (3) non-musicians had lower corticospinal excitability when listening to high-groove music than when they listened to low-groove music or noise, even though they subjectively rated the high-groove clips as higher groove; and (4) non-musicians had higher pre-pulse electromyogram activity when listening to high-groove music than when listening to low-groove music.

In (Matthews et al., 2020), when musicians and non-musicians listened to rhythms of differing degrees of groove, the musicians showed greater activity in certain regions of the brain associated with beat-based timing. These included regions of the brain "associated with beat perception, including the caudate, right prefrontal cortex, SMA [supplementary motor area], primary and premotor cortex and primary and secondary auditory regions" (Matthews et al., 2020). It therefore appears "that musical training leads to greater engagement of regions involved in beat perception as well as stronger auditory-motor associations" (Matthews et al., 2020). 


\subsection{Dance performance}

In this article, the term "dance performance" includes the usual sense of a planned performance of either amateur or professional dancers for an audience. It is also used in a wider sense to denote the physical activity of dancing in any context.

There are a great many difficulties associated with neuroscientific research on dance performance-for more detail, see section 2. The principal challenge is technological, as dance requires the person being scanned to move. EEG headsets and fNIRS allow for investigation of the neural activity of people moving, but have low spatial resolution in the brain. Techniques with higher spatial resolution, such as fMRI and MEG, do not allow for movement.

To work around these difficulties, some research has been carried out on people performing activities which are similar to dancing, but are of such a nature that the participants can be scanned. The neural activity associated with performing these activities is then used as a proxy for neural activity while dancing. We therefore include research on these activities in this section.

We also include the results of a study in which dance-naïve participants learned a modern jazz dance routine, then underwent EEG scanning immediately before and after performing the routine (Wind et al., 2020). Although neural activity was not measured during the dance performance itself, the results have clear relevance to the neuroscience of dance performance, and the study is included both in this section and in the numerical data concerning dance performance studies.

The subjects of the studies in which the neuroscience of dance performance was investigated directly were: (1) performing tango steps lying supine in a scanner; (2) taking part in a dance video game; (3) performing 'expressive' dance movements; and (4) the modern jazz study mentioned above.

\subsubsection{Tango steps lying supine in a scanner}

In (Brown et al., 2006), dancers performed tango steps on an inclined surface while lying supine in a PET scanner. When they did this: (1) the anterior cerebellar vermis was involved in the entrainment of dance steps to music, compared to self-paced movement without music; (2) the right putamen was involved in dancing to a regular metric rhythm, compared to an irregular rhythm; and (3) the medial superior parietal lobule was involved in spatial guidance of leg movements. 


\subsubsection{Dance video game}

In (Ono et al., 2014, 2015; Tachibana et al., 2011), in which fNIRS was used, dancers took part in a dance video game based on Dance Dance Revolution ${ }^{\mathrm{TM}}$. When they did this: (1) activation in the superior parietal lobule and superior temporal gyrus increased as a function of task difficulty (Tachibana et al., 2011); (2) after training, the mean oxygenated haemoglobin waveform changed from activation above baseline to below baseline (Ono et al., 2015); and (3) frontotemporal oxyhaemoglobin dynamics predicted performance accuracy (Ono et al., 2014).

These results seem to indicate that, as the dancers became more adept at playing the dance video game, they might have been guided more by stimulus-led bottom-up cortical activation than by cognition-led top-down cortical activation.

\subsection{3 "Expressive" dance movements}

In (Cruz-Garza et al., 2014), in which EEG was used, dancers performed a range of prescribed "expressive" dance movements. When they did this: "bilateral premotor and motor cortices and dorsal parietal lobule areas" were all involved in these movements. It is not clear from the article, however, whether the effect was bilateral in the premotor and motor cortices or only in the premotor cortex.

While the results are of interest, and "show the feasibility of inferring the expressive component of movements [...] from scalp EEG" (Cruz-Garza et al., 2014), the sample size was small $(n=4)$. It is therefore not possible to generalise the results of this study.

\subsubsection{Scanning before and after performance of a modern jazz dance routine}

In (Wind et al., 2020) dance-naïve participants learned a modern jazz dance routine, and underwent EEG scanning immediately before and after performing the routine. The routine was performed both in the presence and absence of music.

After the performance in the presence of music there was, compared to pre-performance: (1) decreased neural activity in the parietal cortex in the gamma band; and (2) increased functional connectivity between frontal and temporal regions in the theta band.

After the performance in the absence of music there was, compared to pre-performance: (1) increased neural activity in frontal and occipital regions in the alpha band; (2) increased 
frequency in frontal, central, temporal, and parietal regions in the alpha band; (3) increased connectivity between the frontal and occipital lobes in the alpha band; (4) decreased connectivity between electrodes in parietal regions in the alpha band; and (5) decreased neural activity in occipital regions in the gamma band.

Both in the presence and in the absence of music, brain activity and functional connectivity increased after the performance compared to pre-performance.

These results may be indicative, in both the presence and absence of music, of the recruitment of working memory, attentional processing and heightened awareness. Furthermore, the results in the absence of music may imply a state of "relaxed wakefulness," leading to deep concentration. These results may also imply reduced demand for visual processing due to the automatic nature of the dance performance and "the transformation of sensory input into motoric output or the emotions caused by expressive dance" (Wind et al., 2020).

While these results are of interest, a small sample size was used $(n=11)$. It is therefore not possible to generalise the results of this study.

\subsection{Observation}

In this article, the term "observation" is used to indicate the active observation of dance performance. The performance can be either live or recorded. Due to experimental constraints, most of the studies reviewed in this section used recorded dance.

In addition to the importance of dance observation itself, it can be, and often is, used as a proxy to study the neural activity of dance performance. The main reason for this is that it is thought that dance observation engages the human mirror system (Gallese et al., 1996; Grèzes and Decety, 2001; Rizzolatti and Sinigaglia, 2010).

The human mirror system is a process in which the brain simulates the performance of observed movements, without conscious imagery. The network of regions of the brain involved in the system is called the action observation network (AON). The human mirror system is engaged by observation of an action, when similar regions of the brain are activated to those activated when that action is performed (Jeannerod, 1994). There appears to be a significant overlap between the cortical circuits involved in movement generation, movement imagery, and movement observation (May et al., 2011; Munzert et al., 2008).

If dance observation does engage the human mirror system, results pertaining to the neuroscience of dance relating to dance observation can also contribute to our knowledge of 
the neuroscience of dance. However, many reservations have been expressed regarding the human mirror system (for an overview, see e.g. (Heyes and Catmur, 2021), so any generalisation of dance observation results to dance performance should be conducted with caution. Indeed, there seems to be some support for scepticism in the results of a study included in this review (Wind et al., 2020), in which the authors found that their "results indicate differences between a physically executed and imagined dance."

However, the difficulties of research into dance performance itself, and the potential for the production of worthwhile, albeit imperfect, results, are presently such that research into dance observation is worth undertaking as a proxy for research into dance performance.

There are several other benefits to researching dance observation. Training is not required, making it easier to find experimental participants and reducing research expense. However, training in dance performance or music performance, as well as exposure to dance observation, affect the neural activity which occurs when people observe dance. Furthermore, a person can observe dance even if he or she is physically unable to undertake it.

For all of these reasons, we therefore include research on dance observation in this review. The subjects of the studies in which the neuroscience of dance observation was investigated were: (1) auditory processing during dance observation; (2) observing live or recorded dance; (3) movement smoothness; (4) aesthetic judgements; and (5) dance training and familiarity of observed dance.

\subsubsection{Auditory processing during dance observation}

In (Reason et al., 2016), a study on dance observation, there was greater inter-subject correlation when dance was observed in the presence of music in a left anterior region of the superior temporal gyrus (STG) involved in complex audio processing. When dance was observed in the absence of music, there was greater inter-subject correlation in the parietal and occipitotemporal cortices and in a posterior region of the STG. The difference in activation of the STG when dance was observed with and without music may be due to sounds made by the dancer being observed, such as the breath, providing "a more salient auditory signal than music in primary auditory regions" of the brain (Reason et al., 2016).

\subsubsection{Observing live or recorded dance}

The neural activity of people when they observe dance appears to vary according to whether the dance being observed is live or recorded. In (Jola et al., 2011), participants watched a full 
dress rehearsal of the ballet "Sleeping Beauty." Corticospinal excitability decreased over the ballet as a whole, as well as within each of the three acts. This might indicate that, even though it could be expected that motor simulation would occur automatically when movements are observed, in fact, "spectators simulate the movements less, the longer they engage emotionally and cognitively with the story-line of the ballet." However, this was a very small-scale study $(n=4)$.

In a larger-scale study $(\mathrm{n}=20)$ (Jola and Grosbras, 2013), the effects of watching three different types of movement (ballet, Indian Bharatanatyam dance, and an active acting control) were investigated and compared, using both live (in-person) and recorded movement. Corticospinal excitability was significantly greater when participants watched dance live than when they watched a recording. Moreover, the differences in neural activity when the three types of movement were observed were only evident when watching the movements live.

The editing style of dance recordings also affects the neural activity of people when they observe dance. In (Herbec et al., 2015), an unedited recording was shot using one static camera, providing a continuous view. An edited recording was shot using several cameras, providing additional close-up views of the upper body and face, or the feet. Similar activity was observed in the occipital and temporal cortices upon observation of both the unedited and edited recordings. However, upon observation of the unedited recording, there was greater inter-subject correlation in the superior temporal gyrus only. Moreover, upon observation of the edited recording, there was greater inter-subject correlation in the postcentral gyrus, lingual gyrus, precentral gyrus, and medial frontal gyrus.

\subsubsection{Movement smoothness}

Movement smoothness when people observe dance appears to affect neural activity. In (Miura et al., 2010), when a humanoid robot was observed performing dance, greater activation was observed in the cortical networks of motion and body-sensitive visual processing areas, related to motion smoothness, with little variation between subjects. Activation was also observed in several cortical networks related to motion smoothness, including the parietofrontal network and the inferior temporal region, with a large degree of variation between subjects.

It seems from these results that: (1) cortical networks are involved in the processing of motion smoothness of expressive body movements; (2) motion-sensitive and body-sensitive 
visual processing areas of the brain are associated with visual perception in the early phases of cognitive processing of movement; and (3) activity in cortical networks involved in understanding the movements "is influenced by personal attitudes about the consciousness of art” (Miura et al., 2010).

\subsubsection{Aesthetic judgements}

When observing dance, it appears that appreciation of the aesthetic aspects of it relies on a large cortico-subcortical network. This includes regions of the brain involved in visual processing, as well as regions involved in the coding of emotional reward, for example the amygdala (fear/disgust), and the nucleus accumbens, insula, and cingulate cortex (pleasure/reward) (Candidi and Aglioti, 2015).

Furthermore, negative correlation has been observed between subjective emotional judgement and activity in the right posterior parietal cortex (Grosbras et al., 2012). Observation of movements that are rated as both difficult to reproduce and aesthetically pleasing has resulted in the greatest activation of the occipitotemporal and parietal regions of the AON (Cross et al., 2011). Other regions of the brain that also appear to be involved are the extrastriate body area (Calvo-Merino et al., 2010), bilateral occipital cortex, and right premotor cortex (Calvo-Merino et al., 2008).

In (Kirsch et al., 2015), familiarity with the dance being observed affected neural activity related to aesthetic judgements. Participants gained the greatest enjoyment from watching dance sequences with which they were more familiar, and neural activity associated with the aesthetic response shifted "from subcortical regions associated with dopaminergic reward processing to posterior temporal regions involved in processing multisensory integration, emotion, and biological motion" (Kirsch et al., 2015).

It therefore seems that there is a "direct link between posterior parietal cortex activity and emotional reaction to dance" (Grosbras et al., 2012), as well as "a possible role of visual and sensorimotor brain areas in an automatic aesthetic response to dance" (Calvo-Merino et al., 2008). These factors may, therefore, go some way to explaining why dance is culturally universal (Adler, 2009; Brown, 1991; Conard et al., 2009; Grafton and Cross, 2008; Levitin et al., 2018; Lomax, 1978; Spencer, 1985), (Grosbras et al., 2012). 


\subsubsection{Dance training and familiarity of observed dance}

The neural activity of people who observe dance can change as a result of dance training. This change differs according to the age of the observer. Changes can occur in: (1) corticospinal excitability; (2) brain wave activity; and (3) neural activity in specific brain regions and networks. The neural activity of people who observe a performance of specific dance choreography also appears to be affected by whether or not the observed choreography has been learnt.

\subsubsection{Corticospinal excitability}

In (Jola et al., 2012), it was found that the corticospinal excitability of people who observed dance was correlated with their dance experience. This could be increased by visual experience alone, i.e. direct motor experience of dance was not required. It is therefore suggested "that spectators covertly simulate the movements for which they have acquired visual experience, and that empathic abilities heighten motor resonance during dance observation" (Jola et al., 2012).

\subsubsection{Brain wave activity}

In two studies (Poikonen et al., 2018a, 2018b), brain wave synchrony across different electrode channels on the participants' scalps was monitored while they observed dance and non-dance movements. The three groups were: (1) dancers; (2) musicians; and (3) a control group who did not have any significant dance or musical training ("non-musicians/dancers").

In (Poikonen et al., 2018b), when the participants observed rapid dance movements, synchrony across different electrodes in the alpha band decreased in all three groups compared to when they observed choreographed, almost motionless, action. In (Poikonen et al., 2018a), when non-dancers observed a nearly motionless dancer performing ordinary, small, movements in silence, synchrony in the theta and gamma bands was greater than when they observed a still figure. When non-dancers observed "vast energetic" dance movements to music, synchrony in the alpha and beta bands decreased.

In two studies (Orgs et al., 2008; Poikonen et al., 2018b), the power of alpha and beta bands was significantly reduced when dancers observed dance compared to when they observed a visual baseline. This difference was not found when dancers observed everyday movements, nor was it found in non-dancers. Moreover, when dancers observed multimodal, naturalistic dance (Poikonen et al., 2018b), theta synchrony in their frontocentral regions was greater than that which occurred when musicians and non-musicians/dancers observed the same dance. In (Di Nota et al., 2017), when ballet dancers observed a familiar ballet 
sequence, they showed higher individual alpha peak frequency, greater alpha desynchronization, and greater task-related beta power than dancers of other genres and nondancers who observed the same sequence.

In (Orlandi et al., 2020b), when dancers and non-dancer controls observed "effortful" ballet steps: both groups had a greater late positivity effect in posterior regions than when they observed and "effortless" ones; frontoparietal regions were more engaged in dancers and occipitotemporal regions were more engaged in controls. A larger P300 was observed in the frontal regions of dancers when they observed effortful movements than in the controls. In (Orlandi and Proverbio, 2019), when the participants observed ballet steps: (1) in both dancers and non-dancer controls, there was a posterior $\mathrm{N} 2$ component, and in the left hemisphere this was larger in dancers than in controls; and (2) in the dancers, there was engagement of bilateral inferior and middle temporal regions, as well as early P2 and greater P300 responses in both hemispheres. In (Orlandi et al., 2017), dancers and non-dancer controls observed novel dance movements choreographed in such a way that the dancers would not have direct motor experience of them, but would nonetheless find them familiar. Observation of these movements resulted in a larger centroparietal N400 effect and a smaller late positivity amplitude, compared to rest, in dancers only. The regions involved were the frontoparietal network, the limbic system, and regions of the brain associated with processing the body and face.

It appears from these results that: (1) both dance training and musical training affect the brain wave activity of people who observe dance; (2) cortical communication is increased in dancers when they watch dance; and (3) this increase is related both to the observation of dance and to complex internal cognitive and emotional processes.

\subsubsection{Activity in specific brain regions and networks}

When movement is observed, it appears that the action observation network (AON) is "preferentially engaged for [...] more familiar movement exercises" (Diersch et al., 2013).

When dance is observed, activity in the AON varies depending on the observer's level of dance experience and dance familiarity (Amoruso et al., 2014; Burzynska et al., 2017a; Calvo-Merino et al., 2005; Cross et al., 2006, 2009b; Gardner et al., 2015; Jang and Pollick, 2011; Jola et al., 2013; Orgs et al., 2008; Pilgramm et al., 2010). Activity in the AON also varies according to the type of familiarity, for example whether it is physical or solely visual (Calvo-Merino et al., 2006, 2005; Cross et al., 2009a; Jang and Pollick, 2011; Jola et al., 2012). This has been found when comparing activity in the AON in dancers observing moves 
they perform to moves they only observe (Calvo-Merino et al., 2006, 2005; Cross et al., 2009a; Jola et al., 2012) and, in (Jang and Pollick, 2011), when comparing activity in the $\mathrm{AON}$ in dancers to non-dancers and non-dancer expert dance viewers. It also appears that the functional connectivity of the AON is altered in dancers (Burzynska et al., 2017a).

In (Gardner et al., 2015), increased activity occurred in the "left middle temporal gyrus, inferior parietal lobule, and inferior frontal gyrus as videos were rated as increasingly familiar" (Gardner et al., 2015). The AON was therefore preferentially engaged on observation of more familiar movements and there was "attenuation of effective connectivity bidirectionally between parietal and temporal AON nodes when participants observed videos they rated as increasingly familiar" (Gardner et al., 2015). These results are supportive of a predictive coding model of the AON.

In several studies, when dancers observed dance, greater activity occurred in various regions of their brains than in those of non-dancers. These regions were: (1) the premotor cortex (PMC) (Calvo-Merino et al., 2006, 2005; Cross et al., 2009b, 2006; Pilgramm et al., 2010); (2) the inferior parietal lobule (Calvo-Merino et al., 2005; Cross et al., 2006); (3) the middle and posterior superior temporal gyrus (Jola et al., 2013); (4) the right primary somatosensory cortex; the right temporoparietal cortex; and (4) the left retrosplenial cortex (Jang and Pollick, 2011).

However, in (Jang and Pollick, 2011), less activity was observed in the PMC in dancers than in non-dancers. When experienced dance viewers observed dance, they, like dancers, had greater activity than non-dancers in the right temporoparietal cortex and left retrosplenial cortex. However, experienced dance viewers had less activity in the right primary somatosensory cortex, and more activity bilaterally in the PMC, than dancers. In the primary somatosensory cortex, this may be caused by overlearning. It may also occur as a result of neurological, or task, constraints. Dance training may therefore result in the reorganisation of the network of brain regions used during dance observation.

When dancers observed movements from their own repertoire, there was more activity in the PMC (Calvo-Merino et al., 2005), especially the right ventral PMC (Cross et al., 2009a), than when they observed movements they did not perform, but with which they had visual familiarity. Moreover, premotor, parietal, and cerebellar activity were all greater when the dancers observed movements of the same style which are usually performed by people of the same sex as the observers (Calvo-Merino et al., 2006). When expert dancers observed familiar dance movements (Calvo-Merino et al., 2005), they showed more activation in 
regions of the brain generally associated with the human mirror system than when they observed unfamiliar dance movements.

In a study on motor experience of dance movements in both dancers and non-dancers (Cross et al., 2006), motor experience of dance movements modulated activity in the inferior parietal lobule and ventral PMC in both groups. This result supports the theory that "motor vocabularies are stored within these two brain regions" (Cross et al., 2006).

It also appears that anticipatory activity in a fronto-parietal-occipital network enables expert dancers to anticipate and discriminate earlier than non-dancers when observing dance (Amoruso et al., 2014), and that dancers have a greater degree of motor simulation when they observe dance than non-dancers do, with stronger de-synchronisation of the motor cortex (Bläsing et al., 2012).

Overall, these studies provide strong evidence that dance training enhances the AON, strengthening the human mirror system. The AON is also associated with empathy (Jospe et al., 2020, 2018), enabling us to infer information about others' actions and intentions (Caspers et al., 2010; Cross et al., 2009b; Gardner et al., 2015), improve prediction of their future behaviour (Blakemore and Frith, 2005; Falck-Ytter et al., 2006; Hamilton, 2013; Hamilton and Grafton, 2006), and plan appropriate responses. These abilities are of prime importance in social interactions and therefore in the formation and maintenance of social groups. It follows that this research supports the idea that dance has had a significant role in enhancing "brain networks that support our ability to understand others, i.e., our interpersonal coordination skills" (Basso et al., 2021).

\subsubsection{Age, and observing learnt choreographies}

Cortical maturity appears to affect the neural activity of people who observe dance. In (Sumanapala et al., 2018), when young adults observed dance after training in the dance observed, they showed more distinct patterns of activity in their visual cortices than adolescents, and in (Diersch et al., 2013), when older adults observed familiar figure skating movements, they showed greater recruitment of visual regions, the hippocampus, and the caudate, than younger adults.

The neural activity of people who observe a performance of a specific choreographed dance also appears to be affected by whether or not the observers have learnt the choreography. The ways in which choreography is learnt, namely the different modes of learning, also seem to affect the neural activity of people who observe a performance of that choreography after they have learnt it (Kirsch et al., 2018; Kirsch and Cross, 2015; 
Sumanapala et al., 2018). Modes of learning used include: (1) visual training (observation only); (2) auditory training (following spoken instructions); and (3) physical training (performing the movements).

In (Sumanapala et al., 2018), the activation of the AON in participants who had learnt the observed actions through visual training only was different from the activation of the AON in people who had learnt them through both visual and physical training. In (Kirsch and Cross, 2015), the more modes were used in learning an action, the greater the activation of the AON when observing it. Moreover, the better the participant was at performing the action, the stronger the neural response, especially in the PMC.

Age and mode of learning also appear to interact. In (Kirsch et al., 2018), younger adults (18-23 years old) and older adults (55-69 years old) were studied observing a performance of a specific choreographed dance before and after learning it, through visual training and physical training. Different patterns of neural activity were observed in the different age groups. When younger adults observed the dance they had learnt, they showed no increased neural activity, but they did show decreased neural activity in several regions of the right hemisphere, including the fusiform gyrus, postcentral gyrus, and middle temporal gyrus. When older adults observed the dance they had learnt, they showed increased activity in the right precuneus, but showed no decreased neural activity.

Differences also arose between the two age groups when they observed performance of a specific choreographed dance if they learnt it through physical training only. When younger adults observed the dance they had learnt, they again showed no increased neural activity. However, they did show decreased neural activity, albeit in different regions: the amygdala and middle occipital gyrus, and in regions associated with sensorimotor processing, including the left paracentral lobule, postcentral gyrus, right precentral gyrus, and inferior temporal gyrus. When older adults observed the dance they had learnt, they again showed increased activity in the right precuneus, but showed decreased activity in the right superior lobule, right inferior parietal lobule, and right thalamus.

Physical training resulted in the greatest difference in neural activity between younger and older adults when they observed the dance after they had learnt it. Both groups showed decreased activation in the left fusiform gyrus, the right inferior temporal gyrus, and the right inferior parietal lobule. However, young adults showed less neural activity than older adults in the left inferior parietal lobule.

It appears from this research that visual and motor regions of the brain contain experiencespecific representations of the motor actions learnt, with a similar pattern of activity observed 
in both age groups, including less recruitment of sensorimotor and higher-order visual association cortices following training in the actions being observed. Moreover, the results indicate that young adults may have processing efficiency gains that older adults lack, and that visual training effects are quite different on a brain level in younger adults than in older adults. This difference in neural activity may be a way of exploiting different mechanisms to compensate for age-related decline in attentional and visual processing.

\subsection{Imagery}

Motor imagery is "the cognitive simulation of an action without actually executing it" (Carey et al., 2019). In this article, we use the term "imagery" to refer to all motor imagery relevant to dance.

Imagery can be either of oneself, or someone else, moving. In the studies cited in this review, it is either stated or implied that the participants are imagining themselves moving. When the imagined action is dance ("dance imagery"), this activity is intimately related to dance observation. When people observe dance, the mirror neuron system often induces unconscious dance imagery. In this section, however, we only review research on conscious dance imagery.

There is significant overlap between the cortical circuits involved in movement generation, movement imagery, and movement observation (May et al., 2011; Munzert et al., 2008). This means that research on dance imagery can also inform our knowledge of the neuroscience of dance performance and dance observation. However, the reservations which have been expressed regarding mirror neuron theory, mentioned in section 4.4 , must be borne in mind when generalising the results of dance imagery to dance performance.

Some of the studies in this section are concerned with non-dance imagery, such as walking, but are nonetheless included because they pertain both to movement imagery and dance, e.g. investigating the effects of dance training on the neural activity of people who imagine non-dance motor activities.

For all of these reasons, we include research on the neuroscience of movement imagery relevant to dance. The subjects of the relevant studies were: (1) imagery with eyes open or eyes closed; (2) scanning before and after imagery of a modern jazz dance routine; (3) dance training or experience; and (4) familiarity with the choreography. 


\subsubsection{Eyes open/eyes closed}

In (Di Nota et al., 2014), alpha power in participants who imagined dance depended on whether their eyes were open or closed. When their eyes were open, alpha power when they were imagining the dance was significantly higher in all areas except the left temporal regions. When the eyes were closed, alpha power was lower relative to the baseline in the occipital and right parietal regions of their brains. Moreover, when the participants' eyes were open, their ability to imagine the dance was decreased and, when their eyes were closed, their ability to do this was increased.

\subsubsection{Scanning before and after imagining a modern jazz dance routine}

In (Wind et al., 2020) dance-naïve participants learned a modern jazz dance routine, and underwent EEG scanning immediately before and after imagining themselves performing the routine. This was done both in the presence and absence of music.

After imagery in the presence of music, there was, compared to the scan before the task: (1) increased neural activity in temporal and parietal regions in the theta band; and (2) increased connectivity between central and parietal regions in the beta band. These results may indicate that, in addition to the conscious perception and processing of music, visualization of body movements in space was also taking place when the dancers were observing the dance.

After imagery in the absence of music, there was, compared to the scan before the task: (1) decreased neural activity in the alpha, beta, gamma, and theta bands, predominantly in frontal, central, and parietal regions; (2) increased connectivity between frontal and temporal regions in the alpha band; (3) decreased connectivity between frontopolar-central, frontalfrontal, and frontal-central regions in the beta band; and (4) decreased connectivity between frontopolar and parietal regions in the gamma band.

While these results are of interest, a small sample size was used $(n=11)$, and it is therefore not possible to generalise the results.

\subsubsection{Dance training or experience}

Both dancers and non-dancers show activation of the AON when they imagine dance (Burzynska et al., 2017a; Hökelmann and Blaser, 2009). However, in many studies, significant differences have also been found between the neural activity of dancers and the neural activity of non-dancers when they imagine dance. 
Following a one week course in which healthy participants learnt to dance the tango (Sacco et al., 2006), neural activity when the participants imagined walking changed after training. Activity increased in premotor areas and the supplementary motor area, and activity decreased in the right posterior region of the brain. It therefore appears that, after training, the role of visual imagery decreased in favour of motor-kinaesthetic imagery.

In (Park et al., 2002), a Salpuri dancer was asked to recall her performance of the dance. Salpuri is a traditional shamanic Korean dance, originally performed to appease evil spirits. When the dancer recalled her performance of the dance, compared to the resting state, there was: (1) increased neural activity in the alpha band; (2) increased neural activity in the theta band in the frontal midline; (3) increased global field power; and (4) decreased generalized frequency and spatial complexity.

In (Olshansky et al., 2015), when an expert break-dancer imagined dance to familiar music, there was less activity in Heschl's gyrus, and more activity in the supplementary motor area, than when the dancer imagined dance to unfamiliar music.

As these studies only present data on one subject, it is not possible to generalise the results. However, several larger-scale studies on dance imagery by expert dancers have been undertaken.

When professional dancers imagined improvised dance (Fink et al., 2009), they had more alpha activity, and exhibited greater right-hemispheric alpha synchronization, than novice dancers. Neither of these effects was observed when they imagined waltz dancing. These results "complement and extend existing findings on the relationship between EEG alpha activity and creative thinking" (Fink et al., 2009).

In (Di Nota et al., 2017), a study on the imagery of non-dance movements, the individual alpha peak frequency of ballet dancers was faster than that of both dancers of other genres and that of non-dancers. All groups showed significant desynchronization relative to the baseline when they imagined non-dance movements, and more desynchronization when they imagined dance movements than when they imagined non-dance movements. It seems from this study that: (1) complex dance imagery "recruits greater cognitive resources" than the imagery of everyday, non-dance, movements; (2) dance imagery may be a more important factor than non-dance imagery "in driving neural plasticity of action networks" (Di Nota et al., 2017); and (3) the results "confirm and extend evidence for experience-dependent plasticity of alpha and beta activity during" motor imagery.

In (Orlandi et al., 2020a), when participants imagined "effortful" and "effortless" ballet steps, maximum peak amplitude in neural activity 400-550 ms after imagery was greater in 
anterior regions of the brain in the dancers than in the non-dancer controls. In controls only, this maximum peak amplitude was more negative in response to effortful than effortless movements. This difference in response to imagination of effortful and effortless movements "also had a frontal distribution in controls and a centroparietal distribution in dancers" (Orlandi et al., 2020a). The controls had more engagement of prefrontal regions than the dancers, while the dancers had more engagement of occipitotemporal and bilateral sensorimotor areas than the controls. It would therefore appear that the controls experienced more cognitive demands than the dancers, and that the imagery of complex actions recruited visuomotor simulation processes in dancers, but not in the controls.

In (Cross et al., 2006), when the participants observed and simulated another dancer's movements, regions of the brain associated with action simulation and action observation were recruited. These regions included: (1) the inferior parietal lobule; (2) cingulate and supplementary motor areas; (3) the superior temporal sulcus; and (4) the PMC. In the inferior parietal lobule and ventral premotor regions, this activity was modulated by dancers' ratings of their ability to perform the observed movements and by their motor experience. This result supports the theory that "motor vocabularies are stored within these two brain regions" (Cross et al., 2006).

In contrast (Jang and Pollick, 2011), when dancers imagined moving dance sequences and static dance postures, they had more activity in the left retrosplenial cortex, and less activity bilaterally in the PMC and the right orbitofrontal cortex, than non-dancers. Dancers also had less activity in the right temporal pole than experienced dance viewers. Like dancers, experienced dance viewers had more activity in the left retrosplenial cortex when they imagined dance than non-dancers. Dancers showed less activity in the right orbitofrontal cortex than non-dancers, and experienced dance viewers showed more activity in this region than non-dancers. Furthermore, experienced dance viewers showed less activity in the right temporal pole than either dancers or non-dancers. It therefore seems to be the case that cognitive processes enhance experienced dance viewers' perception of dance actions, but not non-dancers.

\subsubsection{Familiarity with the choreography}

In several studies (Bar and DeSouza, 2013, 2016; DeSouza and Bar, 2012; Di Nota et al., 2016), as specific dance choreography was learnt, imagining it resulted in different neural activity in the brain. Throughout a 34 -week study, professional ballet dancers learnt specific 
dance choreography and underwent fMRI scanning while imagining the dance movements they were learning, with musical accompaniment. When they were imagining the movements, activation in a network of brain regions, including the bilateral auditory cortex and supplementary motor area, initially increased (7 weeks from start) then decreased (34 weeks from start) (Bar and DeSouza, 2013, 2016; DeSouza and Bar, 2012). However, this reduction was not observed in the basal ganglia (caudate nucleus). It may be, therefore, that the basal ganglia were involved when the participants were learning these complex motor sequences (Bar and DeSouza, 2013).

Changes in activation also occurred in sub-regions of the lateral occipitotemporal cortex, specifically: (1) the lateral occipital cortex; (2) the extrastriate body area; and (3) the MT+ motion area (this area overlaps substantially with the extrastriate body area) (Di Nota et al., 2016). All three sub-regions were activated the most when observing dance, compared to imagery or movement, and all were activated bilaterally during the viewing task at the end of the 34 weeks (Di Nota et al., 2016). Significant increases in activation throughout the study were observed only in the left lateral occipital cortex and only when the choreography that had not been learnt was being imagined (Di Nota et al., 2016).

Overall, neural activity increased initially as the choreography was learnt, then decreased, possibly as a result of overlearning. This pattern follows the typical inverted-U shape expected as learning takes place. It may therefore be possible to determine the timing of learning sensorimotor tasks using neural imaging (Bar and DeSouza, 2013). However, several of these studies used a small sample ((Bar and DeSouza, 2013) $n=11$, (Bar and DeSouza, 2016; DeSouza and Bar, 2012) n =10), so their results are not generalisable.

\subsection{Dance training}

In the preceding sections, we dealt principally with research on the neural activity of people when they were carrying out a dance-related activity. In this section, however, we deal principally with research on neurological changes which occur after a period, whether short or long, of learning to dance. Over the long term, of course, learning to dance is inseparable from dance performance, so the neurological effects of long-term dance training and performance cannot be separated. We therefore include research on dance training in the review.

Both short- and long-term dance training lead to significant changes in brain structure and neural activity, including, in particular, neural activity during dance performance, imagery, 
and dance observation. We have already reviewed research on the effect of dance training on such neural activity in each of the relevant sections.

In this section, we review studies concerned with the effects of dance training on: (1) resting state neural activity in the brain; (2) neural activity in the brain during a musical recall task; (3) brain structure; (4) corticomuscular coherence; and (5) performing bimanual motion in a leading/following/mutual adaptation paradigm. The studies on bimanual motion are included in this section because they are concerned with neural activity associated with the development of skills specific to leading and following in partner dancing.

\subsubsection{Resting state neural activity}

Resting state neural activity in the brain is affected by dance training. In one small-scale study ((Barnstaple and DeSouza, 2019), $\mathrm{n}=10)$, participation in as little as a one-hour dance class modulated resting state alpha activity in the frontal cortex.

In (Ermutlu et al., 2015), comparing dancers, fast ball sports athletes and controls, the dancers had significantly higher amplitudes in the alpha and beta bands compared to fast ball sports athletes and significantly higher amplitudes in the alpha band compared to controls.

In ( $\mathrm{Lu}$ et al., 2018), expert ballroom dancers had lower amplitude of low-frequency fluctuation in the left lingual gyrus and increased amplitude of low-frequency fluctuation than novice dancers in many regions of the brain, including: (1) the left middle temporal gyrus; (2) the bilateral precentral gyrus; (3) the bilateral inferior frontal gyrus; (4) the left postcentral gyrus; (5) the left inferior temporal gyrus; (6) the right middle occipital gyrus; (7) the right superior temporal gyrus; and (8) the left middle frontal gyrus. More neural activity was observed in sensorimotor regions in expert dancers than in novice dancers. As might be expected from the extra training they have undergone, the expert dancers had more neural activity in sensorimotor regions than novice dancers, as well as "functional alterations in frontal-temporal and frontal-parietal connectivity" (Lu et al., 2018). These changes may well reflect the learning of skills needed for dancing, such as "action perception, attentional control, and movement adjustment" (Lu et al., 2018).

\subsubsection{Neural activity during a musical recall task}

In (Jin et al., 2021), the researchers found that "the dancers were more extraverted, indicative of a heightened need for arousal, exhibited a preference for faster musical tempo, and exhibited elevated EEG beta power during the musical recall task relative to non-dancers. 
Paradoxically, dancers also showed elevated introversion (i.e., melancholic score) relative to non-dancers."

The researchers state, however, that the apparent paradox is explicable by interactional personality theory. It may be that, as a result of their elevated introversion, dancers "seek elevated stimulation and, thereby, choose to engage with active and energetic rhythmic auditory stimulation, thus providing the nervous system with the requisite stimulation for desired arousal." The researchers conclude that dancers "need more stimulation and choose active and energetic music (faster tempo), providing the nervous system with higher arousal levels" (Jin et al., 2021).

\subsubsection{Changes in brain structure following dance training}

Brain structure can also be affected by dance training. Although it appears that total brain volume is unaffected by dance training (Burzynska et al., 2017a), several studies indicate that both white matter and grey matter volumes in various regions of the brain are affected by it (Burzynska et al., 2017a; Dordevic et al., 2018; Hänggi et al., 2010; Meier et al., 2016; Nigmatullina et al., 2015).

In (Hänggi et al., 2010), dancers had lower white matter volume than non-dancers in: (1) both corticospinal tracts; (2) both internal capsules; (3) the corpus callosum; and (4) the left anterior cingulum. In this study, the correlation between volume changes in different regions of the brain and the age at which dance training began was also investigated. Negative correlation was observed between the age at which dance training started and: (1) white matter volume in the internal capsule; and (2) grey matter volume in the right PMC. Positive correlation was observed between the age at which dance training started and white matter volume in: (1) the left precentral gyrus; and (2) the corpus callosum.

In (Nigmatullina et al., 2015), fractional anisotropy in the white matter underlying the left and right PMC was lower in dancers than in an active control group.

In (Hüfner et al., 2011), in which grey matter in the hippocampus was investigated, compared to slackliners (people who walk "along a rope or a narrow piece of strong fabric that is stretched above the ground between two points, less tightly than a tightrope" (Cambridge Dictionary, 2021)), dancers had: (1) lower grey matter volumes in the anterior hippocampal formation and parts of the parietoinsular vestibular cortex; and (2) higher grey matter "volumes in the posterior hippocampal formation and the lingual and fusiform gyri bilaterally" (Hüfner et al., 2011). Moreover, there was: (1) negative correlation between the 
amount of time spent training and grey matter volume in the right anterior hippocampal formation; and (2) positive correlation between the amount of time spent training and grey matter volume in the right posterior hippocampal formation. The lower volume in the right anterior hippocampal formation may be a result of long-term suppression of destabilising vestibular input, while the higher volume in the posterior hippocampal formation may be a result of a greater use of visual cues for balance. It would also therefore appear that there is "spatial separation of vestibular and visual processes in the human hippocampus" (Hüfner et al., 2011).

In (Burzynska et al., 2017a), dancers had lower grey matter volume than non-dancers in several regions of the brain, including: (1) the left PMC; (2) the supplementary motor area; (3) the putamen; and (4) the superior frontal gyrus. In addition, dancers had lower anisotropy in the corticospinal tract than non-dancers. In (Dordevic et al., 2018), dancers had higher grey matter volume in several regions of the brain than non-dancers, including: (1) the right hippocampus; (2) the parahippocampal gyrus; (3) the insula; and (4) the cingulate motor cortex. Variations in grey matter volume also occurred bilaterally within the cerebellum. In (Meier et al., 2016), dancers had: (1) more grey matter volume than handball players in regions of the brain involved in the control of the feet; and (2) lower fractional anisotropy than handball players in the fibres connecting regions of the brain involved in the control of foot and hand areas.

It also appears that diffusivity and coherence in the brain are affected by dance training. In (Giacosa et al., 2016), dancers had more diffusivity and less coherence in sensorimotor pathways than musicians, and in (Giacosa et al., 2019) dancers had greater radial diffusivity than musicians, especially in descending motor pathways. This occurred in all regions of the brain, but most notably in the right hemisphere, and was also negatively correlated with the age at which dance training started (Giacosa et al., 2019).

Cortical thickness also appears to be affected by dance training (Karpati et al., 2017, 2018; Porat et al., 2016). In (Porat et al., 2016), dancers had a trend-level significantly thinner cortex than non-dancers. In (Karpati et al., 2017), dancers and musicians both had greater cortical thickness than controls in superior temporal areas. The same authors (Karpati et al., 2018) found less correlation between cortical thickness in the left dorsolateral prefrontal cortex and mean cortical thickness across the whole brain in dancers than in controls. This lower correlation was associated with better performance on a dance video game task, implying that the dorsolateral prefrontal cortex was structurally decoupled in dancers and 
might have been more affected by local training-related factors than other regions of the brain.

Altered functional connectivity has also been found in dancers in several studies (Burzynska et al., 2017a; Hackney and Earhart, 2009; Li et al., 2015; G. Li et al., 2019). In ( $\mathrm{Lu}$ et al., 2018), expert ballroom dancers had altered functional connectivity between the inferior frontal gyrus and temporal/parietal regions, but novice dancers did not. In (Burzynska et al., 2017a), dancers had greater functional connectivity between the thalamus and the frontal pole. This increased thalamofrontal functional connectivity was positively correlated with dance skill. In the same study, dancers had "enhanced connections of the AON to regions involved in somatosensory association (supramarginal gyrus) and sensory interpretation and body image" (inferior parietal lobule). Dancers also had attenuated connections of the AON to regions of the brain involved in: (1) face and body recognition (fusiform gyrus); (2) visual perception of the human body and parts of the body (lateral occipitotemporal cortex/extrastriate body area); and (3) visual perception (occipital pole).

In ( $\mathrm{Li}$ et al., 2015), the researchers compared the functional connectivity density of dancers with that of non-dancers. The dancers had significantly greater functional connectivity than the controls in: (1) the precentral gyri; (2) postcentral gyri; and (3) bilaterally in the putamen. The dancers also had greater connectivity than the controls between: (1) the middle cingulate cortex and the bilateral putamen; and (2) the precentral and the postcentral gyri ( $\mathrm{Li}$ et al., 2015). These results indicate that the dancers had "enhanced functional integration in the cortico-basal ganglia loops that govern motor control and integration" (Li et al., 2015). In (G. Li et al., 2019), dancers and musicians both had enhanced function of the insular subnetwork, associated with empathic ability, relative to the mean of a large random sample. The enhanced functions included "interoceptive sensorimotor representation (the [posterior insula] subnetwork), affective evaluation (the [ventral anterior insula] subnetwork), and higher-level cognitive control (the [dorsal anterior insula] subnetwork)" (G. Li et al., 2019).

It has been suggested (Karpati et al., 2017) that the changes observed in diffusivity coherence in sensorimotor pathways in dancers arise from greater efficiency in the crossing of fibre pathways. The changes in functional connectivity which were observed in several studies (Burzynska et al., 2017a; Hackney and Earhart, 2009; Li et al., 2015; G. Li et al., 2019) may reflect: (1) the improved empathic ability of dancers; (2) improved sensorimotor function; and (3) facilitation of the integration of internal and external information. 
Overall, it appears that long-term dance training is associated with changes in brain structure, notably cortical thickness and plasticity of grey matter and white matter, especially in regions of the brain associated with motor and auditory functions. This is important as neuroplasticity plays a major role in recovery from brain injury. Neuroplasticity may also reverse adverse effects associated with neurodegenerative diseases (Nahum et al., 2013) and cognitive decline due to old age occurs in conjunction with grey matter atrophy (Ramanoël et al., 2018). These results are therefore highly relevant to the use of dance in dance therapy, discussed in more detail in section 4.7.

\subsubsection{Corticomuscular coherence}

Corticomuscular coherence appears to be affected by dance training. In a study of ballet dancers, weightlifters, and controls (Ushiyama et al., 2010), there was significantly greater coherence in the most distal muscles of the control group than in their more proximal muscles. In both the ballet dancers and weightlifters, less coherence was found than in the control group, especially in the muscles of the lower limbs. It would therefore appear that oscillatory coupling between the sensorimotor cortex and motor neurons can be affected by long-term training. Given the marked differences between weightlifting and dancing, and the similar results found for both groups, it seems that the difference in coherence observed did not relate specifically to dance but to regular, strenuous, physical activity in general.

\subsubsection{Performing bimanual motion in a leading/following/mutual adaptation paradigm}

Many styles of dance, such as tango, waltz, and salsa, are partner dances in which two people dance exclusively together. In such dances, one dancer "leads," directing the movements of both people. The other "follows," moving in response to the leader's non-verbal cues. The skills required to achieve the excellent coordination required are therefore very important to research in the field more broadly. Unfortunately, researchers wishing to investigate these skills face similar, and sometimes greater, challenges to those encountered in investigations of the neuroscience of dance performance. However, experiments have been devised which allow some neuroscientific investigation of these phenomena by investigating limited hand and wrist movements.

In three studies (Chauvigné et al., 2018, 2017; Chauvigné and Brown, 2018), dancers skilled in leading and following roles were recruited. Each participant, while lying supine in a fMRI scanner, engaged in bimanual contact with an experimenter who stood outside the bore 
of the scanner. The experimenter was experienced in both leading and following in partner dancing. The participant and experimenter performed movements of the wrist and metacarpophalangeal joints. The movements were controlled in such a way that the participant always had to move his/her hands actively. The three conditions investigated were: (1) the participant leading and the experimenter following; (2) the participant following and the experimenter leading; and (3) mutual adaptation.

In (Chauvigné and Brown, 2018), neural activity during leading and following was enhanced by prior expertise in the respective roles. Moreover, task-specific regions of the brain tended to be positively correlated with the level of expertise in the role.

In (Chauvigné et al., 2018), neural activity associated with: (1) leading involved regions of the brain "involved in motor planning, navigation, sequencing, action monitoring, and error correction"; (2) following involved regions of the brain "involved in somatosensation, proprioception, motion tracking, social cognition, and outcome monitoring"; and (3) mutual adaptation involved "greater activity in brain areas involved in mentalizing and social reward than" either leading or following.

In (Chauvigné et al., 2017), contrary to the researchers' expectations, more activity occurred in the motion area MT+/V5 when dancers followed than when they led. This region is usually deemed to be responsible for processing visual motion. However, on the basis of these results, the authors suggest that this region of the brain "responds to motion based on force-cues during joint haptic interaction" (Chauvigné et al., 2017), especially when a follower is responding to such cues from a leader.

It therefore appears that leading and following are associated with activity in different regions of the brain and that training in one role can selectively enhance role-related brain activations. Moreover, regions of the brain associated with both motor planning and working memory were found to be important in motor improvisation. There may therefore be distinct advantages to learning both leading and following roles, especially in a therapeutic context.

\subsection{Dance therapy}

Dance therapy is a type of movement therapy based on dance. It builds on all of the areas discussed so far, applying them in a clinical context.

Many health conditions can be positively affected by dance therapy. Notable examples are Parkinson's Disease (Barnstaple and DeSouza, 2019; Batson et al., 2014; Leisman and Aviv, 2020; Shanahan et al., 2015), stroke, multiple sclerosis, Huntington's chorea (Patterson et al., 
2018), traumatic brain injury (Kullberg-Turtiainen et al., 2019), neurodegeneration due to age (Burzynska et al., 2017b; Müller et al., 2017; Zilidou et al., 2018) and various mental health conditions (Barnstaple and DeSouza, 2019; Millman et al., 2020) such as depression and some psychoses (Koch et al., 2014; Lee et al., 2015; Malá et al., 2012; Margariti et al., 2012; Martin et al., 2016; Ventouras et al., 2015).

Dance therapy can also produce positive effects on social, intellectual, emotional, and motor functions (Palo-Bengtsson et al., 1998), reduce stress (Hanna, 2017), increase levels of serotonin (Christensen et al., 2017), and help to develop new neural connections (Brown et al., 2006).

While dance therapy is of course not a magical cure-all, it is a very important application of dance, and we include research on it in this review. Many studies have been carried out on its efficacy. For reviews see, e.g., (Mandelbaum and Lo, 2014; Kshtriya et al., 2015; Millman et al., 2020). However, relatively few studies have been carried out on the neurological changes associated with it. The studies that have been carried out are principally concerned with dance therapy for older adults (10 studies out of the 15 reviewed in this section) rather than addressing specific health conditions. There is therefore a great deal of scope for further research in the latter area.

The subjects of the studies in which the neuroscience of dance therapy was investigated were: (1) potential benefits for specific health conditions; and (2) potential benefits for older adults.

\subsubsection{Potential benefits for specific health conditions}

Some studies have been undertaken on the neuroscientific aspect of dance therapy for individuals suffering from specific health conditions. However, two of the studies collected neuroscientific data for only one participant (Batson et al., 2014; Kullberg-Turtiainen et al., 2019). The others were also conducted on a small scale ((Barnstaple and DeSouza, 2019) n = 4, (Margariti et al., 2012) $n=11$, (Ventouras et al., 2015) $n=8$ ). Therefore, while the results of these studies are promising, a great deal more research is necessary to draw meaningful and reliable conclusions.

In a study on dance therapy for Parkinson's Disease (Batson et al., 2014), after the dance intervention the participant had significantly increased network connectivity between the basal ganglia and cortical areas involved in motor function, especially the PMC. In a study of an individual who had suffered a traumatic brain injury (Kullberg-Turtiainen et al., 2019), 
three subnets of the default mode network showed recovery after the dance intervention. The clearest improvement was observed in the right occipito-parieto-temporal subnet.

In individuals suffering from depression, participation in a one-hour dance class modulated resting state alpha activity in the frontal cortex (Barnstaple and DeSouza, 2019). In a depressive/psychotic psychiatric population, resting state activity in the alpha state increased following the dance intervention (Margariti et al., 2012). In individuals suffering from schizophrenia, a potentiation effect (the persistent strengthening of synapses following recent activity) was observed after the dance intervention in: (1) inter-hemispheric connectivity in frontal areas; (2) the attenuation of inter-hemispheric connectivity of left frontal and right central areas; and (3) bilaterally in the intra-hemispheric connectivity of frontal and central areas (Ventouras et al., 2015).

\subsubsection{Potential benefits for older adults}

In several studies, the effects of dance therapy have been investigated in older adults. The results of these studies are positive on physical measures such as balance tests and cognitive tests, as well as containing relevant neuroscientific results.

In (Zilidou et al., 2018), compared to an active control group, those who undertook the dance intervention had: (1) improved optimal brain performance; (2) better information flow; and (3) functional reorganisation of network nodes.

In (Qi et al., 2019), participants with mild cognitive impairment who underwent dance therapy were compared to a "no-change" control group, who received only their usual care. After three months, those in the dance group "exhibited significantly increased [amplitude of low-frequency fluctuation] in several areas, including the bilateral fronto-temporal, entorhinal, anterior cingulate and parahippocampal cortex" compared with the baseline (Qi et al., 2019).

In (Sejnoha Minsterova et al., 2020), compared to a "no-change" control group that did not take part in any dance intervention, the researchers "detected associations [in healthy older adults after the dance intervention] between improved physical fitness and changes in diffusion tensor imagining measures in the whole white matter skeleton and the corticospinal tract and the superior longitudinal fascicle despite the fact that no significant differences in changes to the [white matter] microstructure were found between the two groups" (Sejnoha Minsterova et al., 2020). 
In (Burzynska et al., 2017b) white matter integrity declined over six months across a majority of regions of the brain in all groups (dance, walking, walking/nutrition, and active control) but, in those undertaking more moderate-to-vigorous physical activity, less decline occurred in prefrontal white matter. Increased fractional anisotropy was observed only in the dance group, where it was observed in the fornix.

As noted in section 4.6.3, dance training also appears to improve neuroplasticity. This is highly relevant for dance therapy because neuroplasticity plays a major role in healing and protecting the brain (Nahum et al., 2013; Ramanoël et al., 2018). Further evidence of the effect of dancing in this regard was found in (Rehfeld et al., 2018), in which only dancing, and not the control activity, was associated with increased levels of neurotrophic factors associated with neuroplasticity, and in (Narici et al., 2017), in which neuromuscular junction damage, as assessed by serum measurements of c-terminal peptide agrin fragment, was reduced by recreational dancing over six months, but not by a control activity.

Increases in brain volume in many regions have also been observed following participation in dance therapy. In (Rehfeld et al., 2017), participants in both the dance and active control groups had increased volume in the hippocampus. Compared to those in the active control group, those in the dance group had: (1) "larger brain volume increases in more areas, including the corpus callosum, cingulate cortex, insula, sensorimotor cortex" (Rehfeld et al., 2018); (2) increased volume in the parahippocampal region (Müller et al., 2017), the left dentate gyrus and the right subiculum (Rehfeld et al., 2017); (3) increased grey matter volume in frontal and temporal regions, including the parahippocampal gyrus and the precentral gyrus (Müller et al., 2016), left precentral gyrus (Müller et al., 2017), and the anterior and medial cingular cortex (Rehfeld et al., 2015); and (4) increased white matter volume in the corpus callosum, and in both the right and left frontal lobes (Rehfeld et al., 2015).

These results are particularly interesting with regard to the older population investigated in these studies, as both grey matter and white matter in various regions of the brain are positively correlated with IQ (Haier et al., 2004, 2010; Schmithorst et al., 2005), and the decline in cognitive function associated with ageing occurs in conjunction with grey matter atrophy (Ramanoël et al., 2018). 


\section{Overview of research to date}

In this section, we provide an overview of the findings discussed in section 4 . We also discuss some general limitations of the studies reviewed and make suggestions for future research.

\subsection{Overview}

We found a great deal of valuable research on the neuroscience of dance. Indeed, there was more material than we expected, with 93 articles published up to and including February 2021 containing original and relevant neuroscientific results. Research in the field started in earnest in 2005, and 61 of the articles reviewed here were published in 2014 or later, out of 92 published since 2005 .

However, the distribution of the research is very uneven. Some areas have received very little attention, with only 3 papers concerned with the neuroscience of groove, 6 with dance performance, and 6 with auditory processing relevant to dance. Other areas, however, have received a great deal more attention, with 35 papers concerned with dance observation, and 54 with dance training.

\subsection{Music/rhythm}

Several motor regions of the brain are engaged when listening to music. This may be a key factor in groove, and therefore in dance, which usually occurs in response to groove. Groove appears to be "driven by a combination of motor and reward regions in the brain" (Matthews et al., 2020), and this may be linked to the long pleasure cycles experienced when dancing.

\subsection{Dance performance}

A few studies have been carried out on the neuroscience of dance performance, and it appears that a network of brain regions is involved in dancing (Karpati et al., 2015). However, the activities used only approximate dance. In addition to the technological constraints discussed in section 2 , there is therefore, as discussed in section 4.4 , some doubt as to how translatable the results are to dance performance (Karpati et al., 2015). 


\subsection{Observation}

Many components of the neuroscience of dance observation appear to be affected by dance training, including corticospinal excitability (Jola et al., 2012), and brain wave activity (Di Nota et al., 2017; Orgs et al., 2008; Poikonen et al., 2018a, 2018b). Dancers also appear to have faster processing and subsequent recognition of dance, with enhanced visuo-motor resonance processes, and refined action processing (Orlandi and Proverbio, 2019; Orlandi et al., 2017, 2020b).

There is strong evidence that dance training enhances the action observation network (AON) (Amoruso et al., 2014; Burzynska et al., 2017a; Calvo-Merino et al., 2005, 2006; Cross et al., 2006, 2009b; Diersch et al., 2013; Gardner et al., 2015; Jang and Pollick, 2011; Jola et al., 2012, 2013; Orgs et al., 2008; Pilgramm et al., 2010). The AON is associated with empathy (Jospe et al., 2020, 2018), enabling us to infer information about others' actions and intentions (Caspers et al., 2010; Cross et al., 2009b; Gardner et al., 2015), improve prediction of their future behaviour (Blakemore and Frith, 2005; Falck-Ytter et al., 2006; Hamilton, 2013; Hamilton and Grafton, 2006) and plan appropriate responses. These abilities are of prime importance in social interactions and therefore in the formation and maintenance of social groups. This supports evolutionary theories of the importance of dance in enhancing "brain networks that support our ability to understand others, i.e., our interpersonal coordination skills" (Basso et al., 2021).

\subsection{Imagery}

Significant differences in neural activity have been found in dancers and non-dancers during motor imagery (Burzynska et al., 2017a; Cross et al., 2006; Di Nota et al., 2017; Fink et al., 2009; Hökelmann and Blaser, 2009; Jang and Pollick, 2011; Olshansky et al., 2015; Park et al., 2002; Sacco et al., 2006). Non-dancers appear to experience greater cognitive demand than dancers (Orlandi et al., 2020a) and dancers recruit more motor-kinaesthetic imagery than visual imagery (Sacco et al., 2006).

Dance imagery also appears to be a greater driver of neural plasticity of action networks than non-dance motor imagery (Di Nota et al., 2017). During imagery of dance choreography that was being learnt, activity initially increased, and subsequently decreased, in a network of brain regions, following the usual pattern of neural activity expected throughout learning (Bar and DeSouza, 2013, 2016; DeSouza and Bar, 2012). 


\subsection{Dance training}

Both short- and long-term dance training lead to significant changes in brain structure (Burzynska et al., 2017a; Dordevic et al., 2018; Giacosa et al., 2016, 2019; Hänggi et al., 2010; Hüfner et al., 2011; Karpati et al., 2017, 2018; G. Li et al., 2019; Li et al., 2015; Meier et al., 2016; Nigmatullina et al., 2015; Porat et al., 2016), as well as in neural activity during, particularly, dance performance, imagery, and dance observation. Dance training also appears to affect resting state neural activity (Barnstaple and DeSouza, 2019; Ermutlu et al., 2015; Lu et al., 2018) and neural activity during musical recall (Jin et al., 2021).

Total brain volume appears to be unaffected by dance training (Burzynska et al., 2017a), but there is evidence that both white matter and grey matter volumes in various brain regions are affected by dance training (Burzynska et al., 2017a; Dordevic et al., 2018; Hänggi et al., 2010; Hüfner et al., 2011; Meier et al., 2016; Nigmatullina et al., 2015). Moreover, it appears that long-term dance training is associated with changes in cortical thickness, as well as plasticity of grey matter and white matter, especially in regions of the brain associated with motor and auditory functions (Burzynska et al., 2017a; Dordevic et al., 2018; Hänggi et al., 2010; Meier et al., 2016; Nigmatullina et al., 2015). As mentioned in sections 4.6.3 and 4.7.2, this is important because of the role played by neuroplasticity in healing and protecting the brain.

\subsection{Dance therapy}

In the majority of the studies on the neuroscience of dance therapy, the potential benefits for older adults have been investigated rather than the potential benefits for specific health conditions. Dance therapy appears to improve optimal brain performance (Zilidou et al., 2018), modulate white matter integrity (Burzynska et al., 2017b), and improve neuroplasticity (Narici et al., 2017; Rehfeld et al., 2018). Increases in volume have also been observed in many regions of the brain following participation in dance therapy (Müller et al., 2016, 2017; Rehfeld et al., 2015, 2017, 2018).

The results of these studies show that, while dance does not offer a panacea for these problems, it does have great therapeutic potential both in age-related cognitive decline and in a broad range of health conditions. 


\subsection{Critique}

The limitations arising from the technical difficulties associated with research on the neuroscience of dance have already been discussed in section 2. Most of the limitations of the individual studies are set out extensively in the articles themselves, so we do not include them here. All studies were carried out in accordance with The Code of Ethics of the World Medical Association (Declaration of Helsinki). Informed consent was obtained for experimentation with human subjects, and data was anonymised. We have remarked on some striking limitations of individual studies in the relevant parts of section 4 , especially when a very small sample size was used $(1 \leq \mathrm{n} \leq 11)$.

One problem in some studies arises from the difficulty of ascertaining whether the results were specifically attributable to dance training, to other trainable, physically demanding activities in general, or to some combination of the two. In other words, the problem is the difficulty of controlling for regular participation in strenuous physical activity.

In several studies, the researchers used one control group of active people, such as basketball players or rowers, without a "no-change" control group. This was the case in several studies on the brain structure of dancers (Ermutlu et al., 2015; Meier et al., 2016; Ushiyama et al., 2010) and in several studies on dance therapy (Müller et al., 2017, 2016; Narici et al., 2017; Rehfeld et al., 2018, 2017, 2015; Zilidou et al., 2018). While this approach may seem sensible, the interpretation of results from such studies is fraught with difficulty, especially if changes over time are investigated. Without a "no-change" control group, it is impossible to determine what changes would have occurred without the intervention, and therefore the exact changes arising from it. This is especially important in studies on dance therapy for older adults, as rapid degeneration can, and often does, occur in the elderly. Indeed, in the two studies on dance therapy for older adults in which a "nochange" control group was included (Qi et al., 2019; Sejnoha Minsterova et al., 2020), differences were observed between the two groups, and in the study that employed multiple different control groups (though none of these groups were a "no-change" group) (Burzynska et al., 2017b), white matter integrity declined in all groups.

Another problem with using only an active control group is the difference in the social aspects of dancing and other physical activities. Taking part in both dance and other physical activities, such as playing basketball or rowing, does have important social aspects. However, their character is very different, both in nature and degree. Furthermore, the social and physical life of people who regularly participate in physical activities such as dance and team sports is very different from the social and physical life of those who do not participate in 
such activities. This difference further complicates attempts to ascertain which results relate specifically to dance rather than to trainable, physically demanding activities in general.

We therefore recommend that, where possible, future studies of this sort include both an active control group and a "no-change" control group. It may also be possible to include a group that builds up social connections, but does not take part in any physically demanding activity. Doing this might make it possible to separate the effects of the physical/exercise aspects of dance from the effects of its social aspects.

Another obstacle to research on the neuroscience of dance therapy is the shortage of participants. This may have contributed to the paucity of research on the neuroscience of dance therapy in individuals suffering from specific health conditions, such as Parkinson's disease or multiple sclerosis.

\subsection{Suggestions for future work}

Much valuable research has been carried out to date. However, as is to be expected in such a new field, much remains to be done. In this section, we identify various gaps in the field, and suggest ways to address them. An important general finding in this regard is that the distribution of the research is very uneven. Some areas have received far more attention than others and one very significant area, the interactive and collective nature of dance, has hardly been researched at all.

\subsubsection{The interactive and collective aspects of dance}

Dance is a highly social activity, with a great deal of interaction between dancers both on and off the dance floor. In partner dances, interaction occurs within the couple, and between couples on the dance floor. In non-partner dances, interaction occurs more loosely between a person and everyone in their immediate vicinity. In group dances, interaction occurs between all of the members of the group. Moreover, groups of dancers very often become friends. Dancing together, and spending time together in classes and at socials, reinforces these connections. The interaction between dancers is therefore a very important aspect of any type of dancing.

However, most research to date has focused on individuals. Very few studies to date have included neuroscientific research on the interactional aspects of dance, and none that we are aware of on the social aspects of dance. The only relevant studies of which we are aware are by Chauvigné et al. (Chauvigné and Brown, 2018; Chauvigné et al., 2017, 2018), but their 
investigations were focused on the specific neural correlates of learning the "leading" and "following" roles of social partner dancing.

We therefore suggest that future research focus on the collective experience of dance, especially aspects relating to communication, social entrainment, and interpersonal synchronization. Since dance is a highly interactive and collective activity, such research is also a powerful tool for studying the predictive and social interaction mechanisms of the brain, as well as aiding in the development of more accurate neuroscientific models of interpersonal interaction.

\subsubsection{Groove}

More research needs to be conducted on the neuroscience of groove directly involving dance. Since groove is a key factor linking music and dance, it would be useful if research in this area were to include both phenomena. While research to date has included both musicians and non-musicians, none of the neuroscientific studies have included dancers. Moreover, none of the studies employ dance, or activities which could be said to approximate dance, such as dance observation.

It would therefore be beneficial to the research areas of both groove and dance to explore this avenue further. For example, similar studies to those already undertaken could be carried out with the addition of a group of dancers, or neural imaging could be undertaken during observation of similar dance choreographies accompanied by high-groove or low-groove music.

\subsubsection{Dance performance}

Although there are still technical difficulties associated with researching dance performance, we suggest that more research be undertaken in this area, possibly using fNIRS, EEG headsets, and Mobile Brain/body Imaging (described in (Barnstaple et al., 2020)). With recent advances in technology, it may also soon be possible to research the neuroscience of dance training in real time, for example by conducting neural scanning during a dance class. At the time of writing (May 2021), there are at least two studies in preparation using new technology, one by Marieke Van Vugt's group at the University of Groningen and the other by José Contreras-Vidal's group at the University of Houston. 


\subsubsection{Observation}

Research on dance observation has mostly used recorded dance. While much dance observation undertaken as a pastime is of recorded dance, such as watching videos online, dance observation also commonly takes place in a live setting. Neural activity during dance observation has been found to depend on whether the dance observed is live or recorded, as well as the editing style of recorded dance (Herbec et al., 2015; Jola et al., 2011; Jola and Grosbras, 2013). More research on the observation of live dance would therefore be valuable. We also suggest that, if recorded dance is used, the editing style must also be taken into account.

\subsubsection{Dance therapy}

The neuroscience of dance therapy has received relatively little attention. This is especially noticeable in the literature concerning specific health conditions. While dance therapy is far from being a panacea, very positive results have been found on physical measures for dance therapy. Moreover, the neuroscientific research that does exist in this area corroborates the results found from physical measures, namely that dance therapy: (1) is an effective method of supporting patients suffering from a broad range of health conditions; (2) has great potential to counteract age-related cognitive decline; and (3) is better suited for counteracting age-related cognitive decline than monotonic physical exercise. We therefore suggest that the neuroscience of dance therapy is a fruitful area for future research.

Further research on the neuroscience of dance generally, as well as dance therapy specifically, could well help to improve the future design and implementation of dance therapy interventions (Millman et al., 2020). Such research could be focused especially on carrying out larger scale studies, as well as investigating a wide range of health conditions. Ideally, comparable dance interventions would be used, as well as including both an active control group and a "no-change" control group.

\section{Conclusion}

The main goal of this article was to provide a comprehensive review of research conducted to date on the neuroscience of dance. We have summarised research from the various research areas or parts of research areas which, in our view, constitute the field, namely: (1) 
music/rhythm; (2) dance performance; (3) observation; (4) imagery; (5) dance training; and (6) dance therapy.

Much valuable research has been carried out to date, but some areas have received far more attention than others. We have therefore made a range of suggestions for future work.

Most research to date has focused on individuals, and we therefore suggest that future research focus on the collective experience of dance, especially aspects relating to communication, social entrainment, and interpersonal synchronization. Other areas that could benefit from further research are groove, dance performance, observation, and dance therapy. We hope that further research in these areas will help to address some of the gaps in the field, and provide stronger links between areas that are currently poorly connected. For example, there are few links between research on groove and the rest of the field. Indeed, even though groove is intimately connected to movement to music, and therefore to dance, no neuroscientific research has been conducted to date on groove directly related to dance.

Further research in the neuroscience of dance can contribute to a broad range of areas, including: (1) prediction in the brain; (2) the underlying brain mechanisms of social interaction, including communication, social entrainment, and interpersonal synchronization; (3) improvisation, using unchoreographed dance; (4) the integration of movement and cognition; (5) movement synchronisation, both to music, and to other people; (6) sensorimotor integration; (7) the effects of long-term, intensive, practice of sensorimotor skills (Karpati et al., 2015); (8) action-perception coupling (Bläsing et al., 2012); (9) advanced motor control; (10) brain plasticity (Karpati et al., 2015); (11) brain-behaviour relationships and their development (Karpati et al., 2015); and (12) neuroaesthetics. Moreover, further research on the neuroscience of dance generally, as well as dance therapy specifically, could well help to improve the future design and implementation of dance therapy interventions (Millman et al., 2020).

\section{Author contributions}

MLK conceived the original idea for the article and provided guidance and comments; PV provided advice and comments; NHDF provided general guidance to OFVE, assisted with the creation of the map and the structuring of the article, advised on drafting and edited the text. He also suggested: the database; the graphical and tabular representation of its data; and the bibliography. OFVE did the initial research, including identifying and contacting researchers in the field, created the map, carried out the literature search, created and updated the 
database and the graphical and tabular representation of its data, identified papers to include in the review, read, summarised and critiqued them, identified lacunae in the research to date and the consequent future research needs and, under the guidance of NHDF, structured the article and did most of the drafting. She also liaised with co-authors and dealt with administrative matters.

\section{Acknowledgements and clarification}

We thank Joana Cabral, José Contreras-Vidal, Falisha Karpati, Jan Stupacher and Marieke van Vugt for their generous assistance, as well as Sofia Martins for providing additional insight based on her own studies and research. We also thank Kevin Henbest, Dario Cattaruzza, Tomáš Halgaš, and Theodor Gescher for their comments in the final stages of drafting.

Nicholas HD Foster and Olivia Foster Vander Elst are father and daughter. Nicholas HD Foster, the co-author of this article, should be distinguished from Nicholas EV Foster, the coauthor of several studies cited herein (Giacosa et al., 2016, 2019; Karpati et al., 2015, 2017, 2018).

\section{Funding}

This research did not receive any specific grant from funding agencies in the public, commercial, or not-for-profit sectors. PV and MLK are supported by the Center for Music in the Brain, funded by the Danish National Research Foundation (DNRF117). MLK is supported by the Centre for Eudaimonia and Human Flourishing funded by the Pettit and Carlsberg Foundations.

\section{Conflicts of interest}

There are no conflicts of interest to declare. 


\section{Appendix: Numerical overview of the studies reviewed}

Studies containing results relevant to music/rhythm in the context of the neuroscience of dance are arranged into categories of auditory processing, and groove. We do not include research on beat perception in the numerical data. Although this research is relevant to the neuroscience of dance, and we include a brief overview of a selection of that research, it is not sufficiently relevant to merit detailed textual or numerical consideration.

The total number of studies published in each area and each year, as well as the total number of unique studies published in each year, are set out in table 2. Data relating to the field as a whole is shown in figure 3. The number of articles published in each area can be seen separately in figures 4-10. The figures are arranged in order of decreasing number of studies.

Table 2: All studies published in each area, arranged by year, listed in order of decreasing number of studies, and total number of unique studies published each year.

\begin{tabular}{|c|c|c|c|c|c|c|c|c|}
\hline & \multicolumn{8}{|c|}{ Number of studies } \\
\hline $\begin{array}{c}\text { Publication } \\
\text { year }\end{array}$ & $\begin{array}{l}\text { Dance } \\
\text { training }\end{array}$ & Observation & Imagery & $\begin{array}{l}\text { Dance } \\
\text { therapy }\end{array}$ & $\begin{array}{l}\text { Auditory } \\
\text { processing }\end{array}$ & $\begin{array}{c}\text { Dance } \\
\text { performance }\end{array}$ & Groove & $\begin{array}{c}\text { Total } \\
\text { unique } \\
\text { studies }\end{array}$ \\
\hline 2002 & & & 1 & & & & & 1 \\
\hline 2003 & & & & & & & & \\
\hline 2004 & & & & & & & & \\
\hline 2005 & 1 & 1 & & & & & & 1 \\
\hline 2006 & 3 & 2 & 2 & & & 1 & & 4 \\
\hline 2007 & & & & & & & & \\
\hline 2008 & 1 & 2 & & & & & & 2 \\
\hline 2009 & 4 & 2 & 2 & & & & & 4 \\
\hline 2010 & 3 & 3 & & & & & & 5 \\
\hline 2011 & 2 & 3 & 1 & & & 1 & & 5 \\
\hline 2012 & 3 & 3 & 1 & 1 & & & & 5 \\
\hline 2013 & 4 & 3 & 1 & & & & 1 & 5 \\
\hline 2014 & 1 & 1 & 1 & 1 & 1 & 2 & & 6 \\
\hline
\end{tabular}




\begin{tabular}{|l|l|l|l|l|l|l|l|l|}
\hline 2015 & 5 & 5 & 1 & 2 & 1 & 1 & & $\mathbf{1 3}$ \\
\hline 2016 & 6 & 1 & 2 & 1 & 2 & & & $\mathbf{8}$ \\
\hline 2017 & 5 & 3 & 2 & 4 & 1 & & 1 & $\mathbf{1 1}$ \\
\hline 2018 & 9 & 4 & & 2 & 1 & & & $\mathbf{1 1}$ \\
\hline 2019 & 4 & 1 & & 3 & & & & $\mathbf{6}$ \\
\hline 2020 & 2 & 1 & 2 & 1 & & 1 & 1 & $\mathbf{5}$ \\
\hline 2021 & 1 & & & & & & & $\mathbf{1}$ \\
\hline Total & $\mathbf{5 4}$ & $\mathbf{3 5}$ & $\mathbf{1 6}$ & $\mathbf{1 5}$ & $\mathbf{6}$ & $\mathbf{6}$ & $\mathbf{3}$ & $\mathbf{9 3}$ \\
\hline
\end{tabular}


(a)

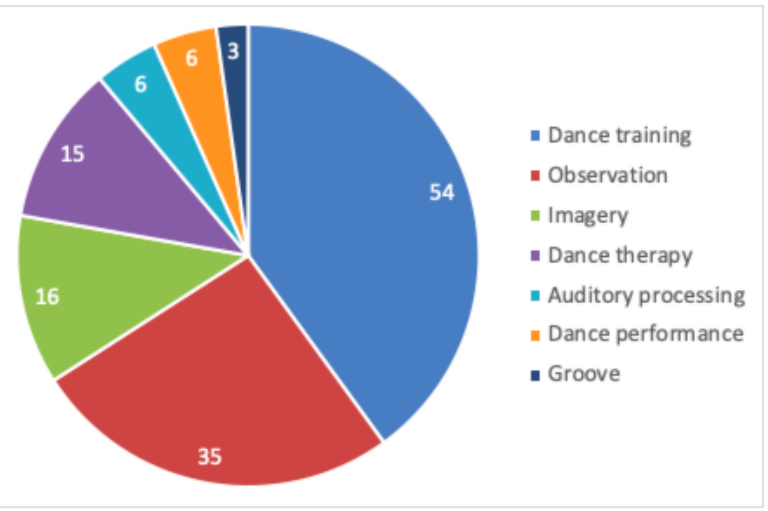

(b)

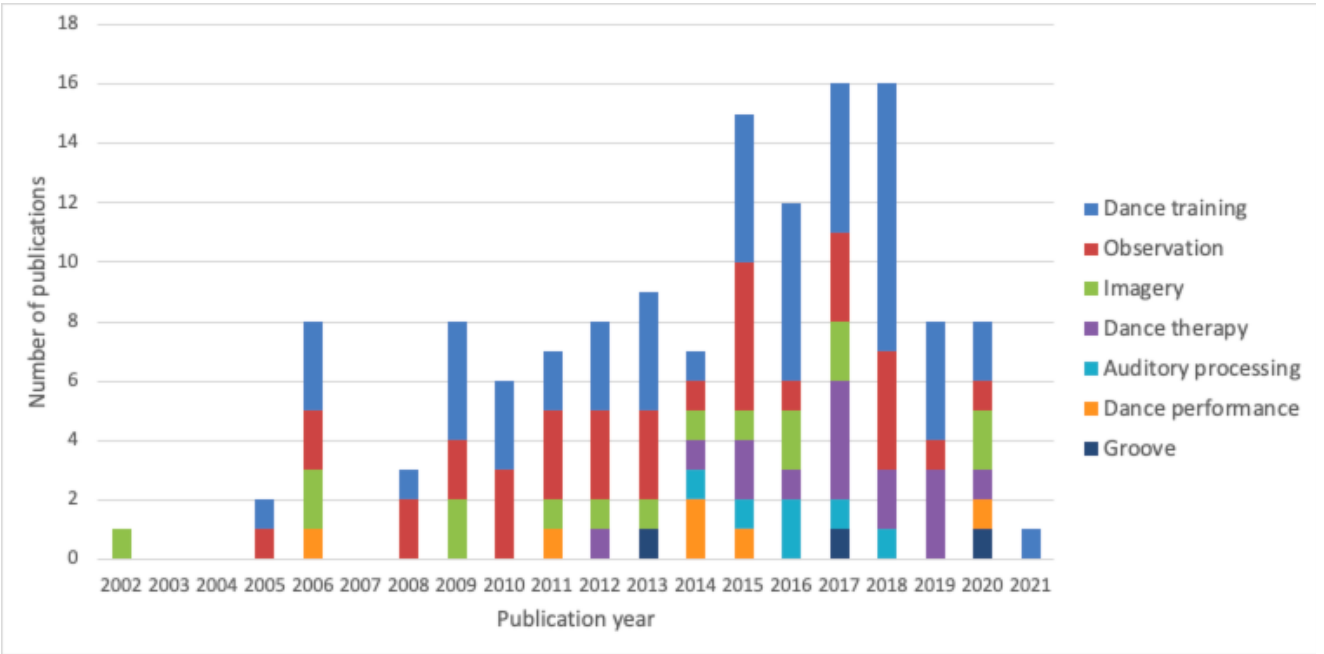

(c)

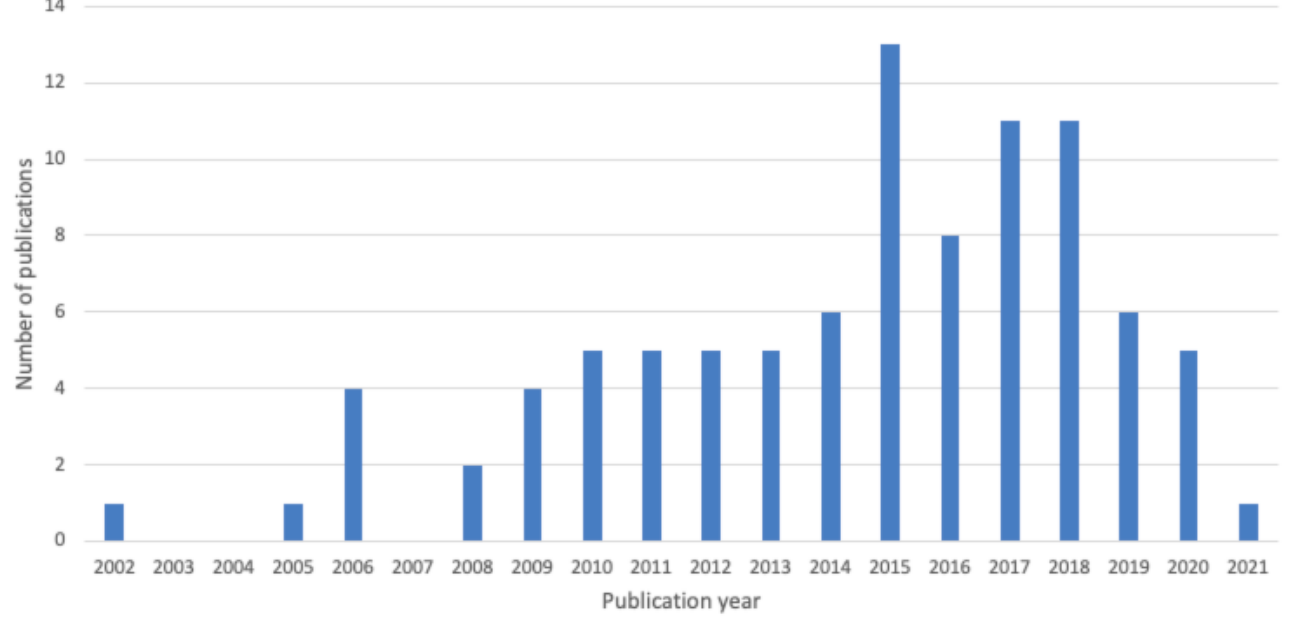

Figure 3: (a) Total number of studies published concerned with each area, listed in clockwise order of decreasing number of studies. (b) Number of studies published each year concerned with each area, listed in order of decreasing number of studies. Some studies are included in more than one area. (c) Total number of individual studies published each year. 
Dance training

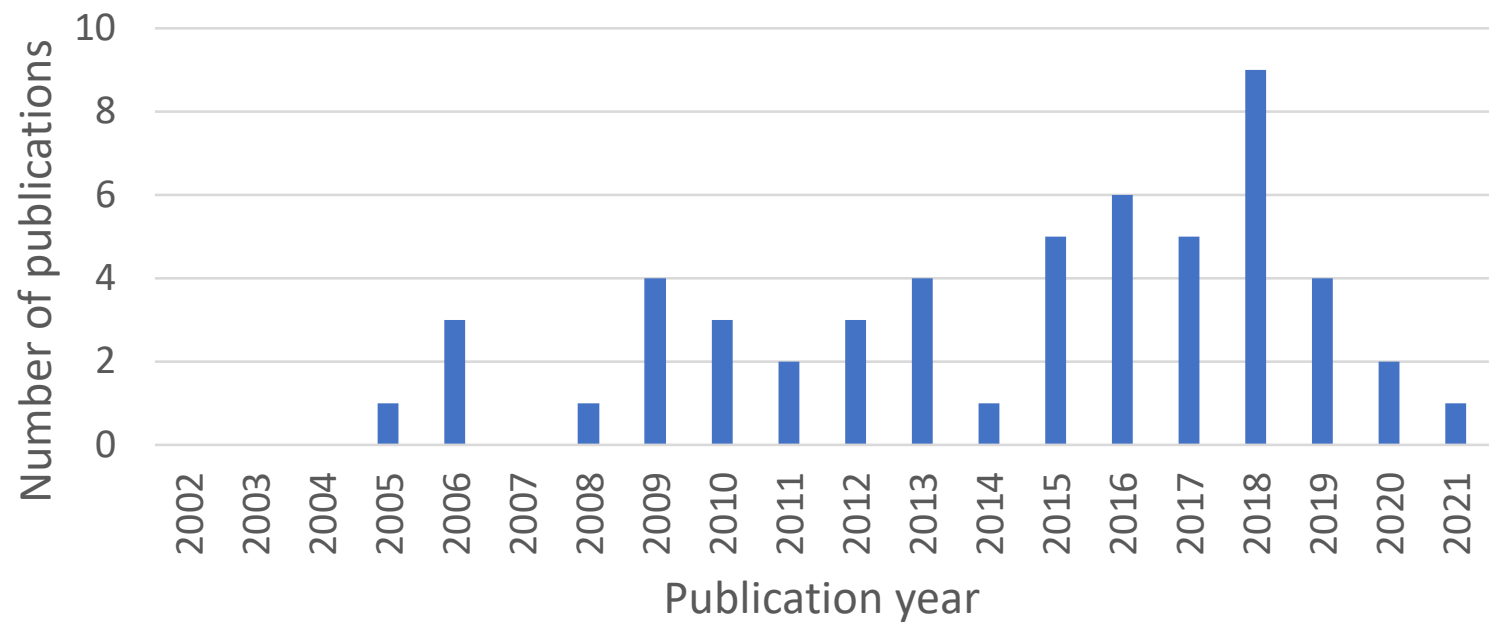

Figure 4: Number of studies published each year concerned with dance training.

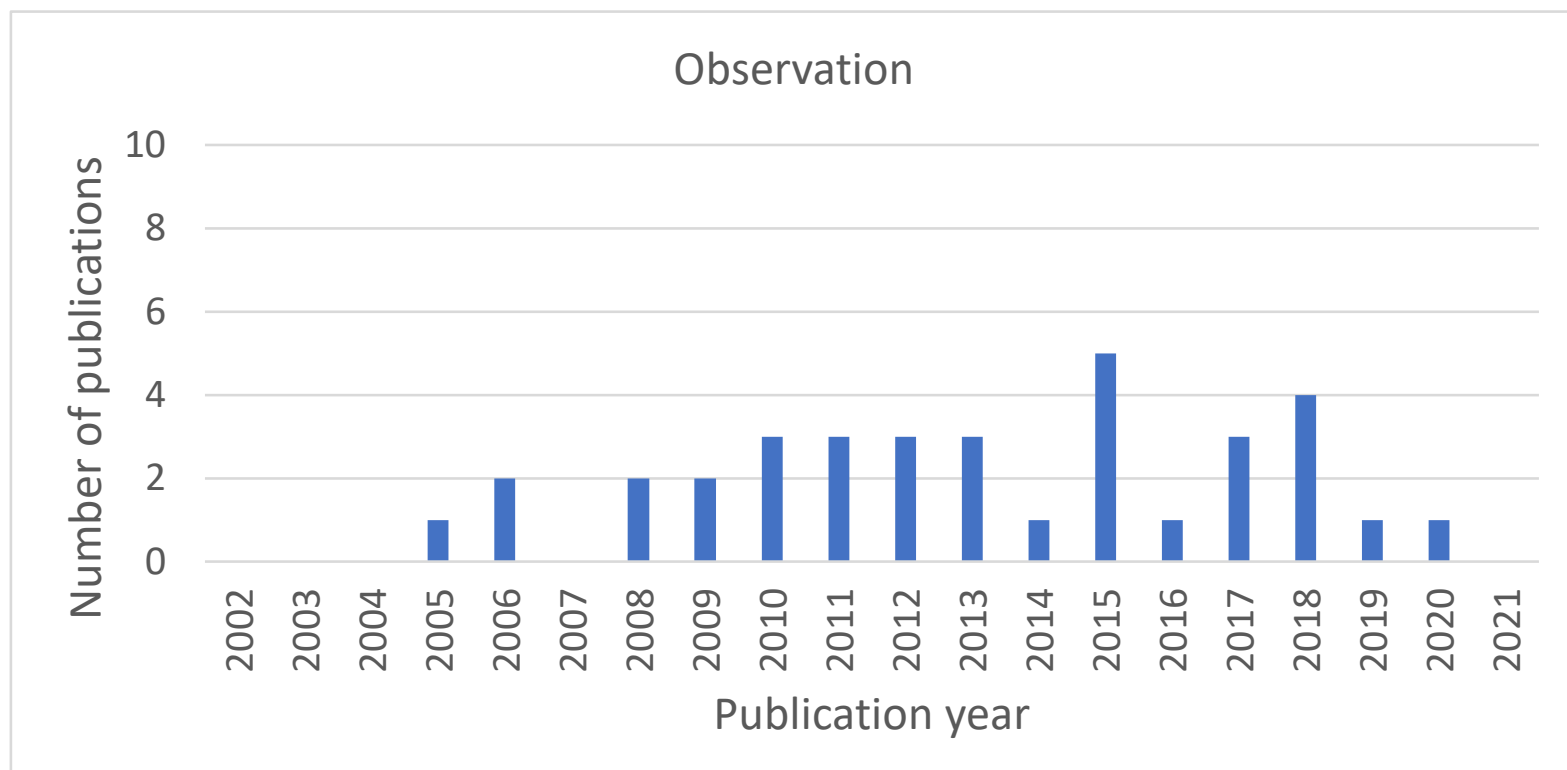

Figure 5: Number of studies published each year concerned with dance observation. 
Imagery

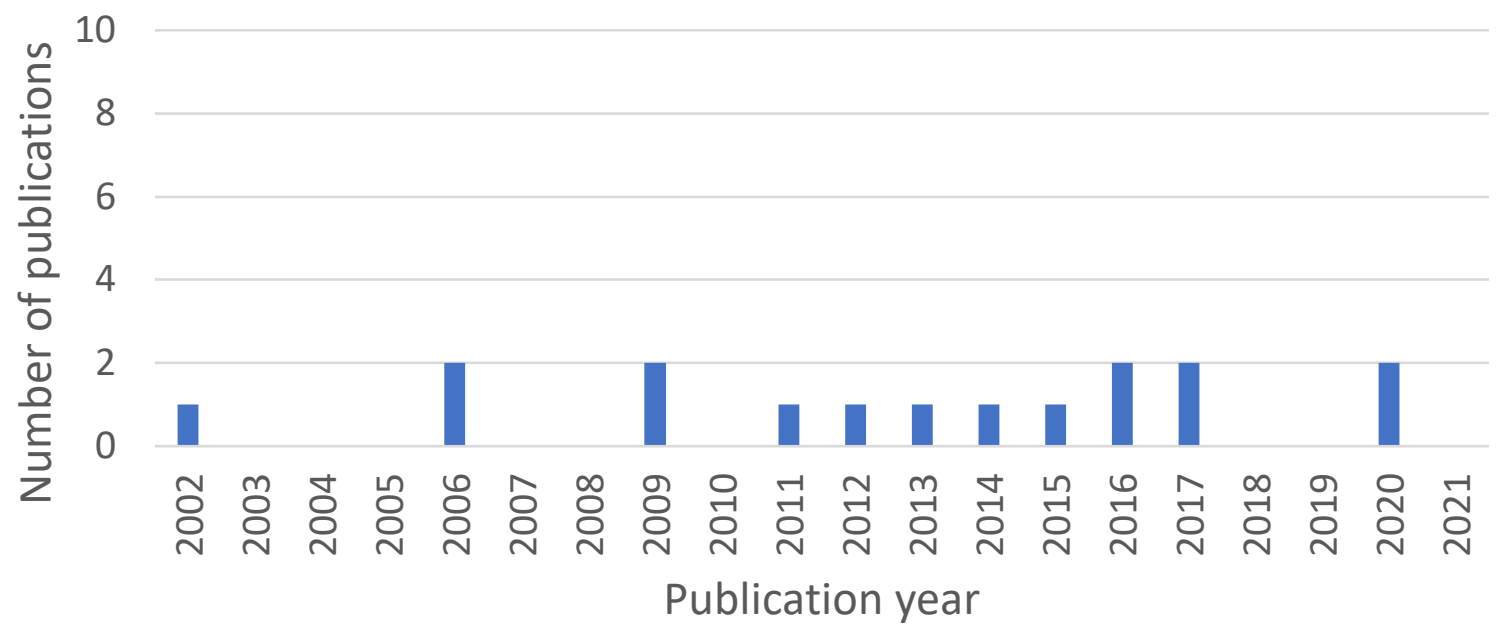

Figure 6: Number of studies published each year concerned with imagery.

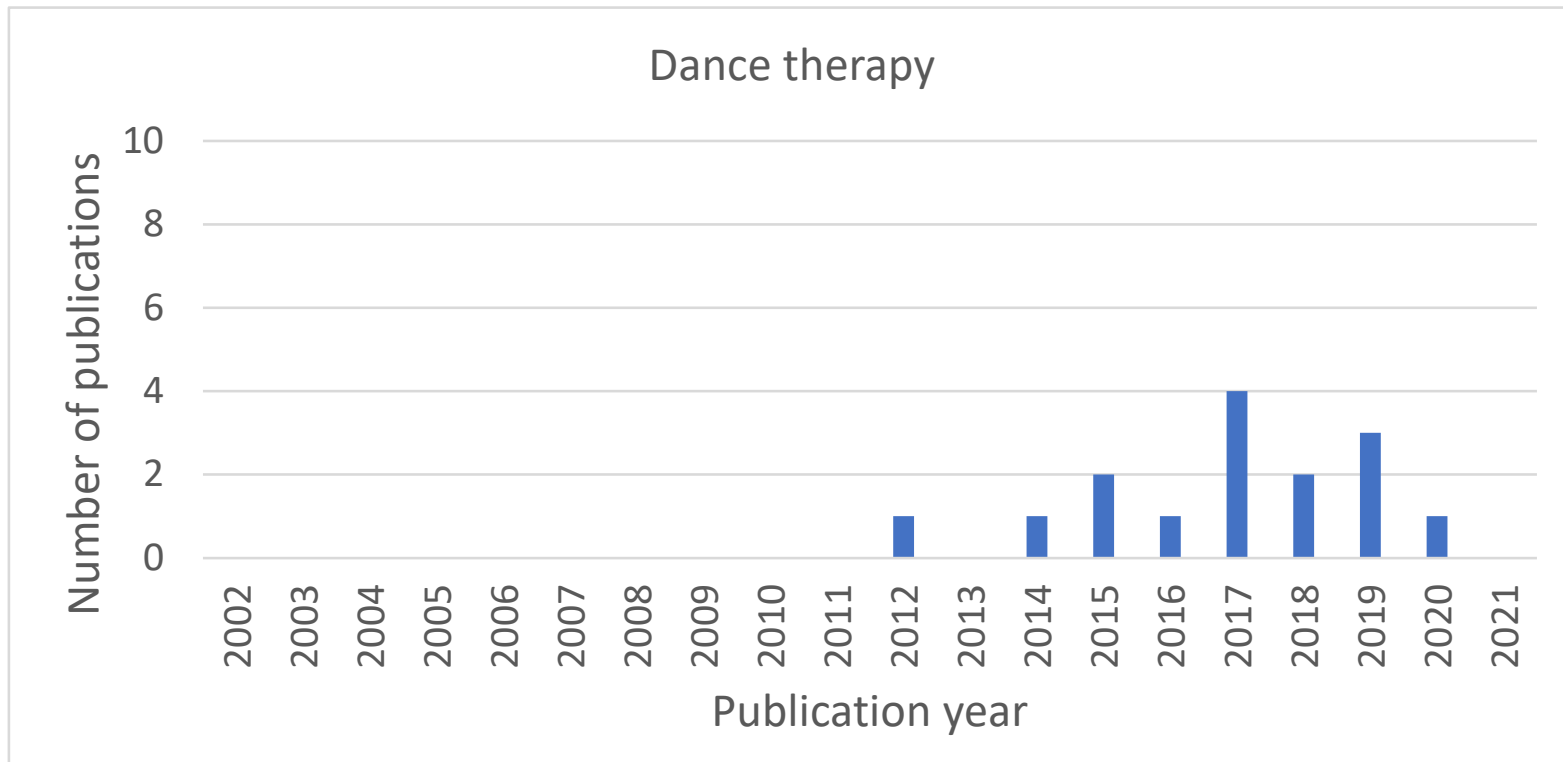

Figure 7: Number of studies published each year concerned with dance therapy. 


\section{Auditory processing}

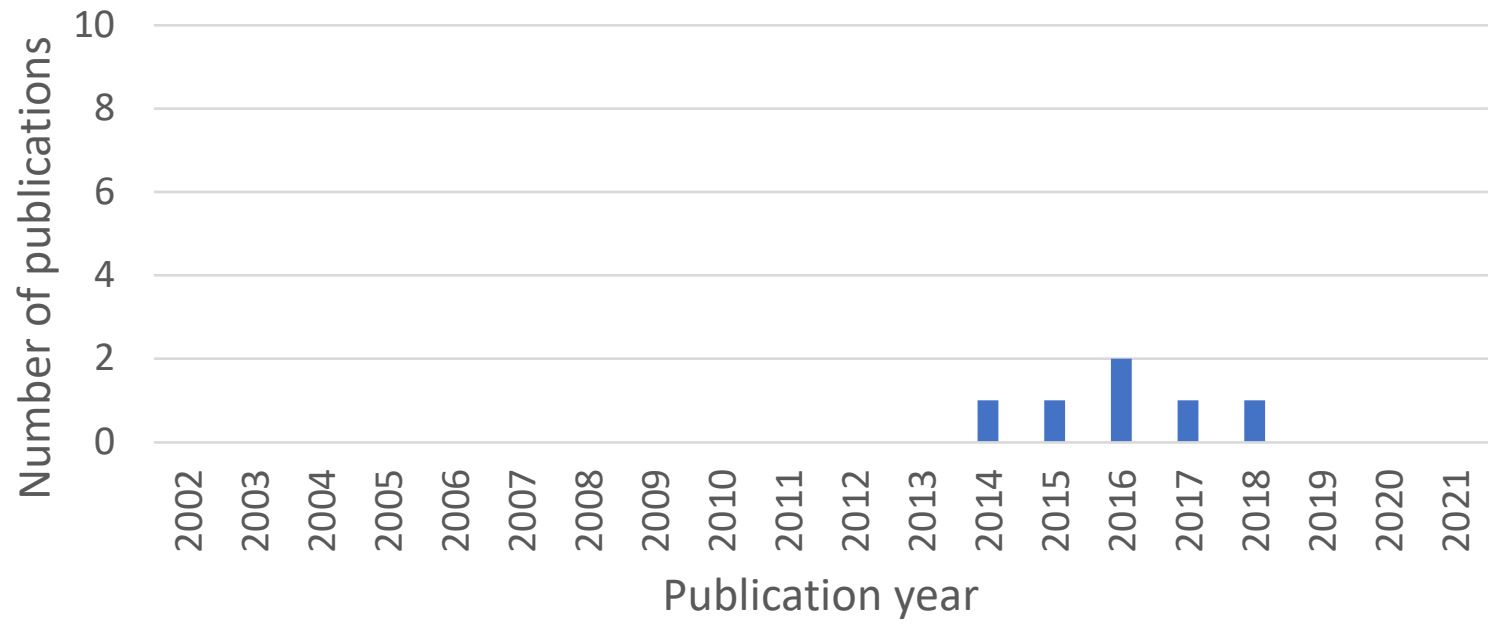

Figure 8: Number of studies published each year concerned with auditory processing of music/rhythm relevant to dance.

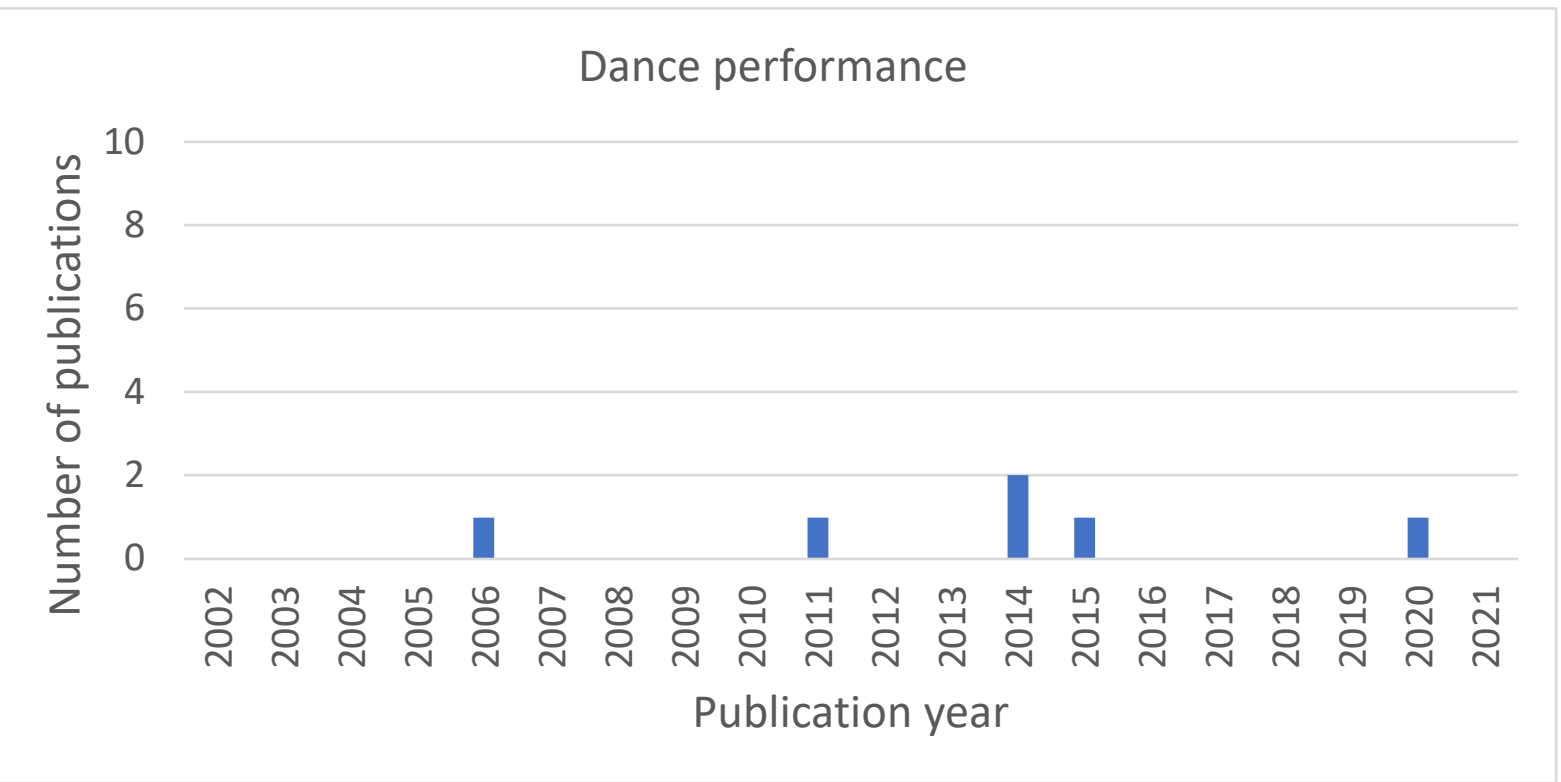

Figure 9: Number of studies published each year concerned with dance performance. 


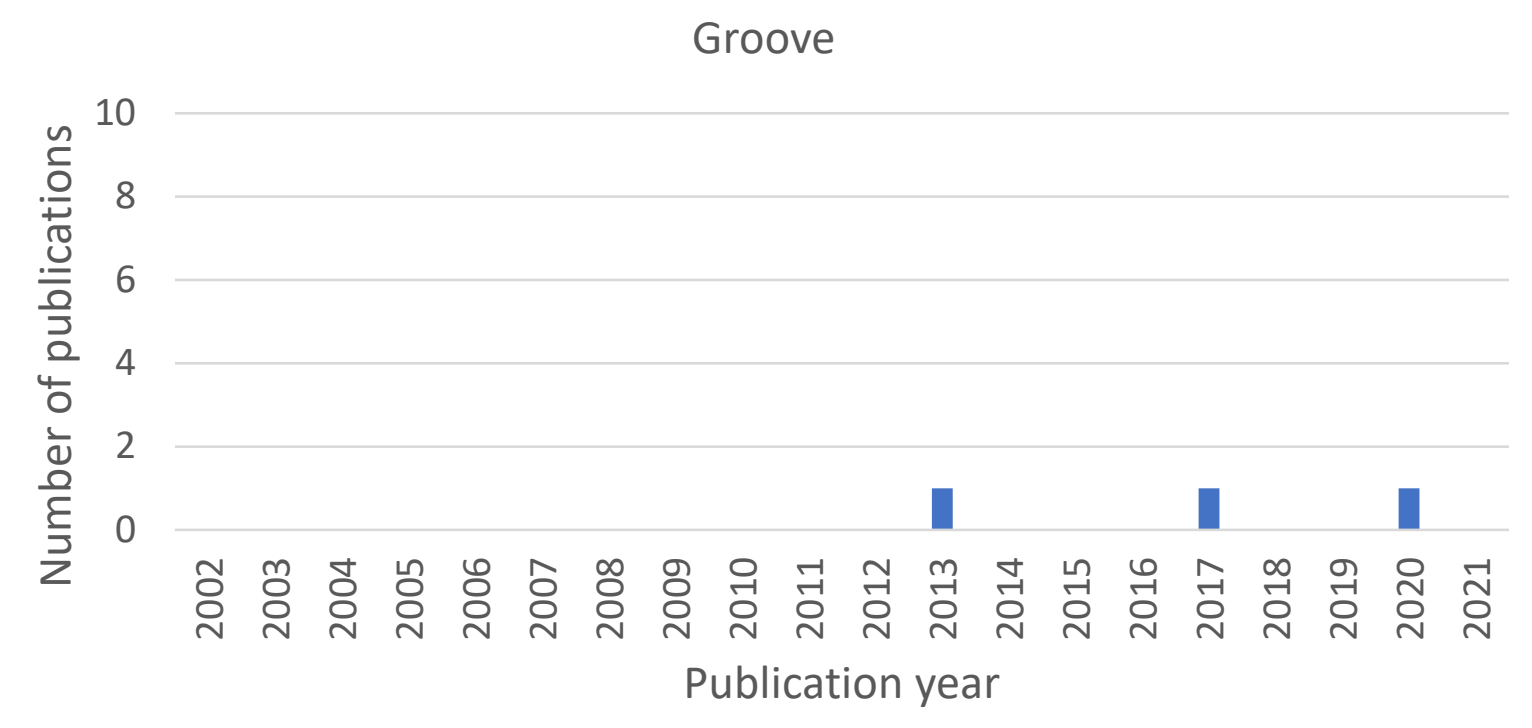

Figure 10: Number of studies published each year concerned with groove.

\section{References}

Adler, D.S., 2009. The earliest musical tradition. Nature 460, 695-696. https://doi.org/10.1038/460695a

Alexander, G.E., DeLong, M.R., Strick, P.L., 1986. Parallel organization of functionally segregated circuits linking basal ganglia and cortex. Annu. Rev. Neurosci. 9, 357381. https://doi.org/10.1146/annurev.ne.09.030186.002041

Amoruso, L., Sedeño, L., Huepe, D., Tomio, A., Kamienkowski, J., Hurtado, E., Cardona, J.F., Álvarez González, M.Á., Rieznik, A., Sigman, M., Manes, F., Ibáñez, A., 2014. Time to tango: expertise and contextual anticipation during action observation. NeuroImage 98, 366-385. https://doi.org/10.1016/j.neuroimage.2014.05.005

Araneda, R., Renier, L., Ebner-Karestinos, D., Dricot, L., Volder, A.G.D., 2017. Hearing, feeling or seeing a beat recruits a supramodal network in the auditory dorsal stream. Eur. J. Neurosci. 45, 1439-1450. https://doi.org/10.1111/ejn.13349

Bar, R.J., DeSouza, J.F., 2013. Tracking the evolution of learning a novel dance sequence in expert ballet dancers. Multisensory Res. 26, 64-64. https://doi.org/10.1163/22134808000S0042

Bar, R.J., DeSouza, J.F.X., 2016. Tracking plasticity: effects of long-term rehearsal in expert dancers encoding music to movement. PLOS ONE 11, e0147731. https://doi.org/10.1371/journal.pone.0147731

Barnstaple, R., DeSouza, J., 2019. Dance and neurorehabilitation - mixed-methods research models. Funct. Neurol. Rehabil. Ergon. 7.

Barnstaple, R., Protzak, J., DeSouza, J., Gramann, K., 2020. Mobile Brain/body Imaging (MoBI) in dance: a dynamic transdisciplinary field for applied research. Eur. J. Neurosci. https://doi.org/10.1111/ejn.14866

Basso, J.C., Satyal, M.K., Rugh, R., 2021. Dance on the brain: enhancing intra- and interbrain synchrony. Front. Hum. Neurosci. 14. https://doi.org/10.3389/fnhum.2020.584312

Batson, G., Migliarese, S.J., Soriano, C., Burdette, J.H., Laurienti, P.J., 2014. Effects of improvisational dance on balance in Parkinson's Disease: a two-phase fMRI case 
study. Phys. Occup. Ther. Geriatr. 32, 188-197. https://doi.org/10.3109/02703181.2014.927946

Bengtsson, S.L., Ullén, F., Henrik Ehrsson, H., Hashimoto, T., Kito, T., Naito, E., Forssberg, H., Sadato, N., 2009. Listening to rhythms activates motor and premotor cortices. Cortex, Special Issue on "The Rhythmic Brain" 45, 62-71. https://doi.org/10.1016/j.cortex.2008.07.002

Berridge, K.C., Kringelbach, M.L., 2015. Pleasure systems in the brain. Neuron 86, 646-664. https://doi.org/10.1016/j.neuron.2015.02.018

Blakemore, S.-J., Frith, C., 2005. The role of motor contagion in the prediction of action. Neuropsychologia, Movement, Action and Consciousness: Toward a Physiology of Intentionality 43, 260-267. https://doi.org/10.1016/j.neuropsychologia.2004.11.012

Bläsing, B., Calvo-Merino, B., Cross, E.S., Jola, C., Honisch, J., Stevens, C.J., 2012. Neurocognitive control in dance perception and performance. Acta Psychol. (Amst.) 139, 300-308. https://doi.org/10.1016/j.actpsy.2011.12.005

Bouwer, F.L., Werner, C.M., Knetemann, M., Honing, H., 2016. Disentangling beat perception from sequential learning and examining the influence of attention and musical abilities on ERP responses to rhythm. Neuropsychologia 85, 80-90. https://doi.org/10.1016/j.neuropsychologia.2016.02.018

Brown, D., 1991. Human Universals. McGraw-Hill Companies,Incorporated.

Brown, S., Martinez, M.J., Parsons, L.M., 2006. The neural basis of human dance. Cereb. Cortex 16, 1157-1167. https://doi.org/10.1093/cercor/bhj057

Burunat, I., Tsatsishvili, V., Brattico, E., Toiviainen, P., 2017. Coupling of action-perception brain networks during musical pulse processing: evidence from region-of-interestbased independent component analysis. Front. Hum. Neurosci. 11. https://doi.org/10.3389/fnhum.2017.00230

Burzynska, A., Finc, K., Taylor, B.K., Knecht, A.M., Kramer, A.F., 2017a. The dancing brain: structural and functional signatures of expert dance training. Front. Hum. Neurosci. 11. https://doi.org/10.3389/fnhum.2017.00566

Burzynska, A., Jiao, Y., Knecht, A., Fanning, J., Awick, E., Chen, T., Gothe, N., Voss, M., McAuley, E., Kramer, A., 2017b. White matter integrity declined over 6-months, but dance intervention improved integrity of the fornix of older adults. Front. Aging Neurosci. 9. https://doi.org/10.3389/fnagi.2017.00059

Calvo-Merino, B., Glaser, D.E., Grèzes, J., Passingham, R.E., Haggard, P., 2005. Action observation and acquired motor skills: an fMRI study with expert dancers. Cereb. Cortex 15, 1243-1249. https://doi.org/10.1093/cercor/bhi007

Calvo-Merino, B., Grèzes, J., Glaser, D.E., Passingham, R.E., Haggard, P., 2006. Seeing or doing? Influence of visual and motor familiarity in action observation. Curr. Biol. 16, 1905-1910. https://doi.org/10.1016/j.cub.2006.07.065

Calvo-Merino, B., Jola, C., Glaser, D.E., Haggard, P., 2008. Towards a sensorimotor aesthetics of performing art. Conscious. Cogn. 17, 911-922. https://doi.org/10.1016/j.concog.2007.11.003

Calvo-Merino, B., Urgesi, C., Orgs, G., Aglioti, S.M., Haggard, P., 2010. Extrastriate body area underlies aesthetic evaluation of body stimuli. Exp. Brain Res. 204, 447-456. https://doi.org/10.1007/s00221-010-2283-6

Cambridge Dictionary, 2021. Definition of "slacklining." Camb. Dict. Online.

Candidi, M., Aglioti, S.M., 2015. Visual and sensorimotor contributions to the esthetic appraisal of body form, motion, and emotion. Eur. Psychol. https://doi.org/10.1027/1016-9040/a000221 
Carey, K., Moran, A., Rooney, B., 2019. Learning choreography: an investigation of motor imagery, attentional effort, and expertise in modern dance. Front. Psychol. 10, 422. https://doi.org/10.3389/fpsyg.2019.00422

Caspers, S., Zilles, K., Laird, A.R., Eickhoff, S.B., 2010. ALE meta-analysis of action observation and imitation in the human brain. NeuroImage 50, 1148-1167. https://doi.org/10.1016/j.neuroimage.2009.12.112

Chapin, H.L., Zanto, T., Jantzen, K.J., Kelso, S.J.A., Steinberg, F., Large, E.W., 2010. Neural responses to complex auditory rhythms: the role of attending. Front. Psychol. 1. https://doi.org/10.3389/fpsyg.2010.00224

Chauvigné, L.A.S., Belyk, M., Brown, S., 2018. Taking two to tango: fMRI analysis of improvised joint action with physical contact. PLoS ONE 13. https://doi.org/10.1371/journal.pone.0191098

Chauvigné, L.A.S., Belyk, M., Brown, S., 2017. Following during physically-coupled joint action engages motion area MT+/V5. J. Integr. Neurosci. 16, 307-318. https://doi.org/10.3233/JIN-170023

Chauvigné, L.A.S., Brown, S., 2018. Role-specific brain activations in leaders and followers $\begin{array}{lllll}\text { during joint action. Front. Hum. Neurosci. } & \end{array}$ https://doi.org/10.3389/fnhum.2018.00401

Chemin, B., Mouraux, A., Nozaradan, S., 2014. Body movement selectively shapes the neural representation of musical rhythms. Psychol. Sci. 25. https://doi.org/10.1177/0956797614551161

Chen, J., Penhune, V., Zatorre, R., 2008. Listening to musical rhythms recruits motor regions of the brain. Cereb. Cortex N. Y. N 1991 18, 2844-54. https://doi.org/10.1093/cercor/bhn042

Christensen, J.F., Cela-Conde, C.J., Gomila, A., 2017. Not all about sex: neural and biobehavioral functions of human dance. Ann. N. Y. Acad. Sci. 1400, 8-32. https://doi.org/10.1111/nyas. 13420

Conard, N.J., Malina, M., Münzel, S.C., 2009. New flutes document the earliest musical tradition in southwestern Germany. Nature 460, 737-740. https://doi.org/10.1038/nature08169

Cross, E.S., Hamilton, A.F. de C., Grafton, S.T., 2006. Building a motor simulation de novo: observation of dance by dancers. NeuroImage 31, 1257-1267. https://doi.org/10.1016/j.neuroimage.2006.01.033

Cross, E.S., Hamilton, A.F. de C., Kraemer, D.J.M., Kelley, W.M., Grafton, S.T., 2009a. Dissociable substrates for body motion and physical experience in the human action observation network. Eur. J. Neurosci. 30, 1383-1392. https://doi.org/10.1111/j.14609568.2009.06941.x

Cross, E.S., Kirsch, L., Ticini, L.F., Schütz-Bosbach, S., 2011. The impact of aesthetic evaluation and physical ability on dance perception. Front. Hum. Neurosci. 5. https://doi.org/10.3389/fnhum.2011.00102

Cross, E.S., Kraemer, D.J.M., Hamilton, A.F. de C., Kelley, W.M., Grafton, S.T., 2009b. Sensitivity of the action observation network to physical and observational learning. Cereb. Cortex 19, 315-326. https://doi.org/10.1093/cercor/bhn083

Cruz-Garza, J.G., Hernandez, Z.R., Nepaul, S., Bradley, K.K., Contreras-Vidal, J.L., 2014. Neural decoding of expressive human movement from scalp electroencephalography (EEG). Front. Hum. Neurosci. 8, 188. https://doi.org/10.3389/fnhum.2014.00188

Dale, J.A., Hyatt, J., Hollerman, J., 2007. The neuroscience of dance and the dance of neuroscience: defining a path of inquiry. J. Aesthetic Educ. 41, 89-110. 
Danielsen, A., Otnæss, M.K., Jensen, J., Williams, S.C.R., Østberg, B.C., 2014. Investigating repetition and change in musical rhythm by functional MRI. Neuroscience $275,469-$ 476. https://doi.org/10.1016/j.neuroscience.2014.06.029

DeSouza, J.F., Bar, R., 2012. The effects of rehearsal on auditory cortex: An fMRI study of the putative neural mechanisms of dance therapy. Seeing Perceiving 25, 45-45. https://doi.org/10.1163/187847612X646677

Di Nota, P.M., Chartrand, J.M., Levkov, G.R., DeSouza, J., 2014. Modulation of alpha power by eye state during kinesthetic motor imagery (KMI) of a newly learned dance sequence in experts. J. Vis. 14, 99-99. https://doi.org/10.1167/14.10.99

Di Nota, P.M., Chartrand, J.M., Levkov, G.R., Montefusco-Siegmund, R., DeSouza, J.F.X., 2017. Experience-dependent modulation of alpha and beta during action observation and motor imagery. BMC Neurosci. 18, 28. https://doi.org/10.1186/s12868-017-03490

Di Nota, P.M., Levkov, G., Bar, R., DeSouza, J.F.X., 2016. Lateral occipitotemporal cortex (LOTC) activity is greatest while viewing dance compared to visualization and movement: learning and expertise effects. Exp. Brain Res. 234, 2007-2023. https://doi.org/10.1007/s00221-016-4607-7

Diersch, N., Mueller, K., Cross, E.S., Stadler, W., Rieger, M., Schütz-Bosbach, S., 2013. Action prediction in younger versus older adults: neural correlates of motor familiarity. PloS One 8, e64195. https://doi.org/10.1371/journal.pone.0064195

Dordevic, M., Schrader, R., Taubert, M., Müller, P., Hökelmann, A., Müller, N.G., 2018. Vestibulo-hippocampal function is enhanced and brain structure altered in professional ballet dancers. Front. Integr. Neurosci. 12. https://doi.org/10.3389/fnint.2018.00050

Ermutlu, N., Yücesir, I., Eskikurt, G., Temel, T., İşoğlu-Alkaç, Ü., 2015. Brain electrical activities of dancers and fast ball sports athletes are different. Cogn. Neurodyn. 9, 257-263. https://doi.org/10.1007/s11571-014-9320-2

Falck-Ytter, T., Gredebäck, G., von Hofsten, C., 2006. Infants predict other people's action goals. Nat. Neurosci. 9, 878-879. https://doi.org/10.1038/nn1729

Fink, A., Graif, B., Neubauer, A.C., 2009. Brain correlates underlying creative thinking: EEG alpha activity in professional vs. novice dancers. NeuroImage 46, 854-862. https://doi.org/10.1016/j.neuroimage.2009.02.036

Foster Vander Elst, O., Vuust, P., Kringelbach, M.L., 2021. Sweet anticipation and positive emotions in music, groove, and dance. Curr. Opin. Behav. Sci. 39, 79-84. https://doi.org/10.1016/j.cobeha.2021.02.016

Fujioka, T., Ross, B., Trainor, L.J., 2015. Beta-band oscillations represent auditory beat and its metrical hierarchy in perception and imagery. J. Neurosci. Off. J. Soc. Neurosci. 35, 15187-15198. https://doi.org/10.1523/JNEUROSCI.2397-15.2015

Gallese, V., Fadiga, L., Fogassi, L., Rizzolatti, G., 1996. Action recognition in the premotor cortex. Brain 119, 593-609. https://doi.org/10.1093/brain/119.2.593

Gardner, T., Goulden, N., Cross, E.S., 2015. Dynamic modulation of the action observation network by movement familiarity. J. Neurosci. 35, 1561-1572. https://doi.org/10.1523/JNEUROSCI.2942-14.2015

Giacosa, C., Karpati, F.J., Foster, N.E.V., Hyde, K.L., Penhune, V.B., 2019. The descending motor tracts are different in dancers and musicians. Brain Struct. Funct. 224, 32293246. https://doi.org/10.1007/s00429-019-01963-0

Giacosa, C., Karpati, F.J., Foster, N.E.V., Penhune, V.B., Hyde, K.L., 2016. Dance and music training have different effects on white matter diffusivity in sensorimotor pathways. NeuroImage 135, 273-286. https://doi.org/10.1016/j.neuroimage.2016.04.048 
Grafton, S., Cross, E.S., 2008. Dance and the brain, in: Gazzaniga, M. (Ed.), Learning, Arts and the Brain. Dana press, New York, pp. 61-70.

Grahn, J.A., Brett, M., 2007. Rhythm and beat perception in motor areas of the brain. J. Cogn. Neurosci. 19, 893-906. https://doi.org/10.1162/jocn.2007.19.5.893

Grahn, J.A., Henry, M.J., McAuley, J.D., 2011. fMRI investigation of cross-modal interactions in beat perception: audition primes vision, but not vice versa. NeuroImage 54, 1231-1243. https://doi.org/10.1016/j.neuroimage.2010.09.033

Grahn, J.A., McAuley, J.D., 2009. Neural bases of individual differences in beat perception. NeuroImage 47, 1894-1903. https://doi.org/10.1016/j.neuroimage.2009.04.039

Grahn, J.A., Rowe, J.B., 2013. Finding and feeling the musical beat: striatal dissociations between detection and prediction of regularity. Cereb. Cortex N. Y. NY 23, 913-921. https://doi.org/10.1093/cercor/bhs083

Grahn, J.A., Rowe, J.B., 2009. Feeling the beat: premotor and striatal interactions in musicians and nonmusicians during beat perception. J. Neurosci. 29, 7540-7548. https://doi.org/10.1523/JNEUROSCI.2018-08.2009

Grahn, J.A., Schuit, D., 2012. Individual differences in rhythmic ability: behavioral and neuroimaging investigations. Psychomusicology Music Mind Brain 22, 105-121. https://doi.org/10.1037/a0031188

Grèzes, J., Decety, J., 2001. Functional anatomy of execution, mental simulation, observation, and verb generation of actions: a meta-analysis. Hum. Brain Mapp. 12, 1-19. https://doi.org/10.1002/1097-0193(200101)12:1<1::AID-HBM10>3.0.CO;2-V

Grosbras, M.-H., Tan, H., Pollick, F., 2012. Dance and emotion in posterior parietal cortex: a low-frequency rTMS study. Brain Stimulat., Human Brain Stimulation in Cognitive Neuroscience 5, 130-136. https://doi.org/10.1016/j.brs.2012.03.013

Haber, S.N., 2003. The primate basal ganglia: parallel and integrative networks. J. Chem. Neuroanat. 26, 317-330. https://doi.org/10.1016/j.jchemneu.2003.10.003

Hackney, M.E., Earhart, G.M., 2009. Effects of dance on movement control in Parkinson's disease: A comparison of Argentine tango and American ballroom. J. Rehabil. Med. 41, 475-481. https://doi.org/10.2340/16501977-0362

Haier, R.J., Jung, R.E., Yeo, R.A., Head, K., Alkire, M.T., 2004. Structural brain variation and general intelligence. NeuroImage 23, 425-433. https://doi.org/10.1016/j.neuroimage.2004.04.025

Haier, R.J., Schroeder, D.H., Tang, C., Head, K., Colom, R., 2010. Gray matter correlates of cognitive ability tests used for vocational guidance. BMC Res. Notes 3, 206. https://doi.org/10.1186/1756-0500-3-206

Hamilton, A.F. de C., 2013. The mirror neuron system contributes to social responding. Cortex 49, 2957-2959. https://doi.org/10.1016/j.cortex.2013.08.012

Hamilton, A.F. de C., Grafton, S.T., 2006. Goal representation in human anterior intraparietal sulcus. J. Neurosci. 26, 1133-1137. https://doi.org/10.1523/JNEUROSCI.455105.2006

Hänggi, J., Koeneke, S., Bezzola, L., Jäncke, L., 2010. Structural neuroplasticity in the sensorimotor network of professional female ballet dancers. Hum. Brain Mapp. 31, 1196-1206. https://doi.org/10.1002/hbm.20928

Hanna, J.L., 2017. Dancing to resist, reduce, and escape stress, in: Karkou, V., Oliver, S., Lycouris, S. (Eds.), The Oxford Handbook of Dance and Wellbeing. Oxford University Press, New York, NY. https://doi.org/10.1093/oxfordhb/9780199949298.013.5

Hanna, J.L., 1987. To Dance is Human: A Theory of Nonverbal Communication. University of Chicago Press, Chicago. 
Haybron, D., 2020. Happiness, in: Zalta, E.N. (Ed.), The Stanford Encyclopedia of Philosophy (Summer 2020 Edition). Metaphysics Research Lab, Stanford University, Stanford.

Heggli, O.A., Konvalinka, I., Kringelbach, M.L., Vuust, P., 2019. Musical interaction is influenced by underlying predictive models and musical expertise. Sci. Rep. 9, 1-13. https://doi.org/10.1038/s41598-019-47471-3

Herbec, A., Kauppi, J.-P., Jola, C., Thoka, J., Pollick, F.E., 2015. Differences in fMRI intersubject correlation while viewing unedited and edited videos of dance performance. Cortex 71, 341-348. https://doi.org/10.1016/j.cortex.2015.06.026

Heyes, C., Catmur, C., 2021. What happened to mirror neurons? Perspect. Psychol. Sci.

Hökelmann, A., Blaser, P., 2009. Mental reproduction of a dance choreography and its effects on physiological fatigue in dancers. J. Hum. Sport Exerc. 4, 129-141. https://doi.org/10.4100/jhse.2009.42.06

Hüfner, K., Binetti, C., Hamilton, D.A., Stephan, T., Flanagin, V.L., Linn, J., Labudda, K., Markowitsch, H., Glasauer, S., Jahn, K., Strupp, M., Brandt, T., 2011. Structural and functional plasticity of the hippocampal formation in professional dancers and slackliners. Hippocampus 21, 855-865. https://doi.org/10.1002/hipo.20801

Janata, P., Tomic, S.T., Haberman, J.M., 2012. Sensorimotor coupling in music and the psychology of the groove. J. Exp. Psychol. Gen. 141, 54-75. https://doi.org/10.1037/a0024208

Jang, S.H., Pollick, F.E., 2011. Experience influences brain mechanisms of watching dance. Dance Res. 29, 352-377. https://doi.org/10.3366/drs.2011.0024

Jarbo, K., Verstynen, T.D., 2015. Converging structural and functional connectivity of orbitofrontal, dorsolateral prefrontal, and posterior parietal cortex in the human striatum. J. Neurosci. 35, 3865-3878. https://doi.org/10.1523/JNEUROSCI.263614.2015

Jeannerod, M., 1994. The representing brain: neural correlates of motor intention and imagery. Behav. Brain Sci. 17, 187-202. https://doi.org/10.1017/S0140525X00034026

Jin, X., Lu, Y., Hatfield, B.D., Wang, X., Wang, B., Zhou, C., 2021. Ballroom dancers exhibit a dispositional need for arousal and elevated cerebral cortical activity during preferred melodic recall. PeerJ 9. https://doi.org/10.7717/peerj.10658

Jola, C., Abedian-Amiri, A., Kuppuswamy, A., Pollick, F.E., Grosbras, M.-H., 2012. Motor simulation without motor expertise: enhanced corticospinal excitability in visually $\begin{array}{lllll}\text { experienced dance } & \text { spectators. } & \text { PLOS } & \text { ONE }\end{array}$ https://doi.org/10.1371/journal.pone.0033343

Jola, C., Grosbras, M.-H., 2013. In the here and now: enhanced motor corticospinal excitability in novices when watching live compared to video recorded dance. Cogn. Neurosci. 4, 90-98. https://doi.org/10.1080/17588928.2013.776035

Jola, C., McAleer, P., Grosbras, M.-H., Love, S.A., Morison, G., Pollick, F.E., 2013. Uniand multisensory brain areas are synchronised across spectators when watching unedited dance recordings. -Percept. 4, 265-284. https://doi.org/10.1068/i0536

Jola, C., Pollick, F.E., Grosbras, M.-H., 2011. Arousal decrease in Sleeping Beauty: audiences' neurophysiological correlates to watching a narrative dance performance of two-and-a-half hours. Dance Res. 29, 378-403. https://doi.org/10.3366/drs.2011.0025

Jospe, K., Flöel, A., Lavidor, M., 2020. The interactive effect of empathy and motor cortex stimulation on hand gesture comprehension. Neuropsychologia 141, 107412. https://doi.org/10.1016/j.neuropsychologia.2020.107412 
Jospe, K., Flöel, A., Lavidor, M., 2018. The interaction between embodiment and empathy in facial expression recognition. Soc. Cogn. Affect. Neurosci. 13, 203-215. https://doi.org/10.1093/scan/nsy005

Karpati, F.J., Giacosa, C., Foster, N.E.V., Penhune, V.B., Hyde, K.L., 2018. Structural Covariance Analysis Reveals Differences Between Dancers and Untrained Controls. Front. Hum. Neurosci. 12. https://doi.org/10.3389/fnhum.2018.00373

Karpati, F.J., Giacosa, C., Foster, N.E.V., Penhune, V.B., Hyde, K.L., 2017. Dance and music share gray matter structural correlates. Brain Res. 1657, 62-73. https://doi.org/10.1016/j.brainres.2016.11.029

Karpati, F.J., Giacosa, C., Foster, N.E.V., Penhune, V.B., Hyde, K.L., 2015. Dance and the brain: a review. Ann. N. Y. Acad. Sci. 1337. https://doi.org/10.1111/nyas.12632

Kirsch, L.P., Cross, E.S., 2015. Additive routes to action learning: layering experience shapes engagement of the action observation network. Cereb. Cortex 25, 4799-4811. https://doi.org/10.1093/cercor/bhv167

Kirsch, L.P., Dawson, K., Cross, E.S., 2015. Dance experience sculpts aesthetic perception and related brain circuits. Ann. N. Y. Acad. Sci. 1337, 130-139. https://doi.org/10.1111/nyas.12634

Kirsch, L.P., Diersch, N., Sumanapala, D.K., Cross, E.S., 2018. Dance training shapes action perception and its neural implementation within the young and older adult brain. Neural Plast. 2018, 5459106. https://doi.org/10.1155/2018/5459106

Koch, S., Kunz, T., Lykou, S., Cruz, R., 2014. Effects of dance movement therapy and dance on health-related psychological outcomes: A meta-analysis. Arts Psychother. 41, 4664. https://doi.org/10.1016/j.aip.2013.10.004

Kringelbach, M.L., Berridge, K.C., 2017. The affective core of emotion: linking pleasure, subjective well-being, and optimal metastability in the brain. Emot. Rev. J. Int. Soc. Res. Emot. 9, 191-199. https://doi.org/10.1177/1754073916684558

Kringelbach, M.L., Berridge, K.C. (Eds.), 2009. Pleasures of the Brain, Series in Affective Science. Oxford University Press, Oxford, New York.

Kshtriya, S., Barnstaple, R., Rabinovich, D.B., DeSouza, J.F.X., 2015. Dance and aging: a critical review of findings in neuroscience. Am. J. Dance Ther. 37, 81-112. https://doi.org/10.1007/s10465-015-9196-7

Kullberg-Turtiainen, M., Vuorela, K., Huttula, L., Turtiainen, P., Koskinen, S., 2019. Individualized goal directed dance rehabilitation in chronic state of severe traumatic brain injury: a case study. Heliyon 5, e01184. https://doi.org/10.1016/j.heliyon.2019.e01184

Kung, S.-J., Chen, J.L., Zatorre, R.J., Penhune, V.B., 2013. Interacting cortical and basal ganglia networks underlying finding and tapping to the musical beat. J. Cogn. Neurosci. 25, 401-420. https://doi.org/10.1162/jocn_a_00325

Launay, J., Tarr, B., Dunbar, R.I.M., 2016. Synchrony as an adaptive mechanism for largescale human social bonding. Ethology 122, 779-789. https://doi.org/10.1111/eth.12528

Lee, H.-J., Jang, S.-H., Lee, S.-Y., Hwang, K.-S., 2015. Effectiveness of dance/movement therapy on affect and psychotic symptoms in patients with schizophrenia. Arts Psychother. 45, 64-68. https://doi.org/10.1016/j.aip.2015.07.003

Leisman, G., Aviv, V., 2020. A neuroscience of dance: potential for therapeusis in neurology, in: Colombo, B. (Ed.), Brain and Art: From Aesthetics to Therapeutics. Springer International Publishing, Cham, pp. 121-138. https://doi.org/10.1007/978-3-03023580-2_10 
Levitin, D.J., Grahn, J.A., London, J., 2018. The psychology of music: rhythm and movement. Annu. Rev. Psychol. 69, 51-75. https://doi.org/10.1146/annurev-psych122216-011740

Lewis, J., 2013. A cross-cultural perspective on the significance of music and dance on culture and society: insight from bayaka pygmies, in: Arbib, M.A. (Ed.), Language, Music, and the Brain: A Mysterious Relationship. The MIT Press, Cambridge, MA, pp. 45-65. https://doi.org/10.7551/mitpress/9780262018104.003.0002

Lexová, I., 2012. Ancient Egyptian Dances. Courier Corporation.

Li, G., He, H., Huang, M., Zhang, X., Lu, J., Lai, Y., Luo, C., Yao, D., 2015. Identifying enhanced cortico-basal ganglia loops associated with prolonged dance training. Sci. Rep. 5. https://doi.org/10.1038/srep10271

Li, G., He, H., Li, X., Zhang, L., Yao, Y., Ye, G., Lu, J., Zhou, S., Yang, L., Luo, C., Yao, D., 2019. Increased insular connectivity and enhanced empathic ability associated with dance/music training. Neural Plast. 2019, e9693109. https://doi.org/10.1155/2019/9693109

Li, Q., Liu, G., Wei, D., Liu, Y., Yuan, G., Wang, G., 2019. Distinct neuronal entrainment to beat and meter: revealed by simultaneous EEG-fMRI. NeuroImage 194, 128-135. https://doi.org/10.1016/j.neuroimage.2019.03.039

Lomax, A., 1978. Folk Song Style and Culture. Taylor and Francis, New York, NY.

Longuet-Higgins, H.C., Lee, C.S., 1984. The rhythmic interpretation of monophonic music. Music Percept. Interdiscip. J. 1, 424-441. https://doi.org/10.2307/40285271

Lu, Y., Zhao, Q., Wang, Y., Zhou, C., 2018. Ballroom dancing promotes neural activity in the sensorimotor system: a resting-state fMRI study. Neural Plast. 2018, 1-7. https://doi.org/10.1155/2018/2024835

Madison, G., 2006. Experiencing groove induced by music: consistency and phenomenology. Music Percept. Interdiscip. J. 24, 201-208. https://doi.org/10.1525/mp.2006.24.2.201

Malá, A., Karkou, V., Meekums, B., 2012. Dance/movement therapy (D/MT) for depression: a scoping review. https://doi.org/10.1016/j.aip.2012.04.002

Mandelbaum, R., Lo, A.C., 2014. Examining dance as an intervention in Parkinson's disease: a systematic review. Am. J. Dance Ther. 36, 160-175. https://doi.org/10.1007/s10465-014-9181-6

Margariti, A., Ktonas, P.Y., Hondraki, P., Daskalopoulou, E., Kyriakopoulos, G.D., Economou, N.-T., Tsekou, H., Paparrigopoulos, T., Barbousi, V., Vaslamatzis, G., 2012. An application of the Primitive Expression form of dance therapy in a psychiatric population. https://doi.org/10.1016/j.aip.2012.01.001

Martin, L.A.L., Koch, S.C., Hirjak, D., Fuchs, T., 2016. Overcoming disembodiment: the effect of movement therapy on negative symptoms in schizophrenia-a multicenter $\begin{array}{lllll}\text { randomized } & \text { controlled } & \text { trial. } & \text { Front. } & \text { Psychol. }\end{array}$ https://doi.org/10.3389/fpsyg.2016.00483

Matell, M.S., Meck, W.H., 2004. Cortico-striatal circuits and interval timing: coincidence detection of oscillatory processes. Cogn. Brain Res., Neuroimaging of Interval Timing 21, 139-170. https://doi.org/10.1016/j.cogbrainres.2004.06.012

Matthews, T.E., Witek, M.A.G., Heggli, O.A., Penhune, V.B., Vuust, P., 2019. The sensation of groove is affected by the interaction of rhythmic and harmonic complexity. PLOS ONE 14, e0204539. https://doi.org/10.1371/journal.pone.0204539

Matthews, T.E., Witek, M.A.G., Lund, T., Vuust, P., Penhune, V.B., 2020. The sensation of groove engages motor and reward networks. NeuroImage 214. https://doi.org/10.1016/j.neuroimage.2020.116768 
May, J., Calvo-Merino, B., DeLahunta, S., McGregor, W., Cusack, R., Owen, A., Veldsman, M., Ramponi, C., Barnard, P., 2011. Points in mental space: an interdisciplinary study of imagery in movement creation. Dance Res. 29, 402-430.

Mcauley, J., Henry, M., Tkach, J., 2012. Tempo mediates the involvement of motor areas in beat perception. Ann. N. Y. Acad. Sci. 1252, 77-84. https://doi.org/10.1111/j.17496632.2011.06433.x

Meier, J., Topka, M.S., Hänggi, J., 2016. Differences in cortical representation and structural connectivity of hands and feet between professional handball players and ballet dancers. Neural Plast. 2016, 6817397. https://doi.org/10.1155/2016/6817397

Merchant, H., Grahn, J., Trainor, L., Rohrmeier, M., Fitch, W.T., 2015. Finding the beat: a neural perspective across humans and non-human primates. Philos. Trans. R. Soc. B Biol. Sci. 370, 20140093. https://doi.org/10.1098/rstb.2014.0093

Merker, B.H., Madison, G.S., Eckerdal, P., 2009. On the role and origin of isochrony in human rhythmic entrainment. Cortex J. Devoted Study Nerv. Syst. Behav. 45, 4-17. https://doi.org/10.1016/j.cortex.2008.06.011

Merriam-Webster.com. 2021. https://www.merriam-webster.com (16 May 2021). https://www.merriam-webster.com/dictionary/war\%20dance

Millman, L.S.M., Terhune, D.B., Hunter, E.C.M., Orgs, G., 2020. Towards a neurocognitive approach to dance movement therapy for mental health: a systematic review. Clin. Psychol. Psychother. https://doi.org/10.1002/cpp.2490

Mithen, S., 2005. The singing Neanderthals: The origins of music, language, mind, and body.

Miura, N., Sugiura, M., Takahashi, M., Sassa, Y., Miyamoto, A., Sato, S., Horie, K., Nakamura, K., Kawashima, R., 2010. Effect of motion smoothness on brain activity while observing a dance: An fMRI study using a humanoid robot. Soc. Neurosci. 5, 40-58. https://doi.org/10.1080/17470910903083256

Moher, D., Liberati, A., Tetzlaff, J., Altman, D.G., Group, T.P., 2009. Preferred Reporting Items for Systematic Reviews and Meta-Analyses: The PRISMA Statement. PLOS Med. 6, e1000097. https://doi.org/10.1371/journal.pmed.1000097

Morillon, B., Baillet, S., 2017. Motor origin of temporal predictions in auditory attention. Proc. Natl. Acad. Sci. 114, E8913-E8921. https://doi.org/10.1073/pnas.1705373114

Müller, P., Rehfeld, K., Hökelmann, A., Kaufmann, J., Mueller, N., 2016. Dance training induced grey matter brain plasticity in the elderly. pp. 117-121.

Müller, P., Rehfeld, K., Schmicker, M., Hökelmann, A., Dordevic, M., Lessmann, V., Brigadski, T., Kaufmann, J., Müller, N.G., 2017. Evolution of neuroplasticity in response to physical activity in old age: the case for dancing. Front. Aging Neurosci. 9. https://doi.org/10.3389/fnagi.2017.00056

Munzert, J., Zentgraf, K., Stark, R., Vaitl, D., 2008. Neural activation in cognitive motor processes: comparing motor imagery and observation of gymnastic movements. Exp. Brain Res. 188, 437-444. https://doi.org/10.1007/s00221-008-1376-y

Nahum, M., Lee, H., Merzenich, M.M., 2013. Principles of neuroplasticity-based rehabilitation, in: Merzenich, M.M., Nahum, M., Van Vleet, T.M. (Eds.), Changing Brains: Applying Brain Plasticity to Advance and Recover Human Ability. Elsevier, Amsterdam; Oxford, pp. 141-171. https://doi.org/10.1016/B978-0-444-633279.00009-6

Narici, M., Rehfeld, K., Mueller, N., Hökelmann, A., 2017. Neuroprotective effects of dancing in old age. Innov. Aging 1, 707-707. https://doi.org/10.1093/geroni/igx004.2532

Nigmatullina, Y., Hellyer, P.J., Nachev, P., Sharp, D.J., Seemungal, B.M., 2015. The neuroanatomical correlates of training-related perceptuo-reflex uncoupling in dancers. Cereb. Cortex. https://doi.org/10.1093/cercor/bht266 
Olshansky, M.P., Bar, R.J., Fogarty, M., DeSouza, J.F.X., 2015. Supplementary motor area and primary auditory cortex activation in an expert break-dancer during the kinesthetic motor imagery of dance to music. Neurocase 21, 607-617. https://doi.org/10.1080/13554794.2014.960428

Ono, Y., Noah, J.A., Zhang, X., Nomoto, Y., Suzuki, T., Shimada, S., Tachibana, A., Bronner, S., Hirsch, J., 2015. Motor learning and modulation of prefrontal cortex: an fNIRS assessment. J. Neural Eng. 12, 066004. https://doi.org/10.1088/17412560/12/6/066004

Ono, Y., Nomoto, Y., Tanaka, S., Sato, K., Shimada, S., Tachibana, A., Bronner, S., Noah, J.A., 2014. Frontotemporal oxyhemoglobin dynamics predict performance accuracy of dance simulation gameplay: temporal characteristics of top-down and bottom-up cortical activities. NeuroImage. https://doi.org/10.1016/j.neuroimage.2013.05.071

Orgs, G., Dombrowski, J.-H., Heil, M., Jansen-Osmann, P., 2008. Expertise in dance modulates alpha/beta event-related desynchronization during action observation. Eur. J. Neurosci. 27, 3380-3384. https://doi.org/10.1111/j.1460-9568.2008.06271.x

Orlandi, A., Arno, E., Proverbio, A.M., 2020a. The effect of expertise on kinesthetic motor imagery of complex actions. Brain Topogr. 33, 238-254. https://doi.org/10.1007/s10548-020-00760-X

Orlandi, A., D'Incà, S., Proverbio, A.M., 2020b. Muscular effort coding in action representation in ballet dancers and controls: electrophysiological evidence. Brain Res. 1733, 146712. https://doi.org/10.1016/j.brainres.2020.146712

Orlandi, A., Proverbio, A.M., 2019. Bilateral engagement of the occipito-temporal cortex in response to dance kinematics in experts. Sci. Rep. 9. https://doi.org/10.1038/s41598018-37876-x

Orlandi, A., Zani, A., Proverbio, A., 2017. Dance expertise modulates visual sensitivity to complex biological movements. Neuropsychologia 104. https://doi.org/10.1016/j.neuropsychologia.2017.08.019

Palo-Bengtsson, L., Winblad, B., Ekman, S.-L., 1998. Social dancing: a way to support intellectual, emotional and motor functions in persons with dementia. J. Psychiatr. Ment. Health Nurs. 5, 545-554. https://doi.org/10.1046/j.1365-2850.1998.560545.x

Park, J.R., Yagyu, T., Saito, N., Kinoshita, T., Hirai, T., 2002. Dynamics of brain electric field during recall of Salpuri dance performance. Percept. Mot. Skills 95, 955-962. https://doi.org/10.2466/pms.2002.95.3.955

Patterson, K.K., Wong, J.S., Prout, E.C., Brooks, D., 2018. Dance for the rehabilitation of balance and gait in adults with neurological conditions other than Parkinson's disease: A systematic Heliyon review. 4 e0584. https://doi.org/10.1016/j.heliyon.2018.e00584

Pilgramm, S., Lorey, B., Stark, R., Munzert, J., Vaitl, D., Zentgraf, K., 2010. Differential activation of the lateral premotor cortex during action observation. BMC Neurosci. 11, 89. https://doi.org/10.1186/1471-2202-11-89

Poikonen, H., Toiviainen, P., Tervaniemi, M., 2018a. Naturalistic music and dance: cortical phase synchrony in musicians and dancers. PLOS ONE 13, e0196065. https://doi.org/10.1371/journal.pone.0196065

Poikonen, H., Toiviainen, P., Tervaniemi, M., 2018b. Dance on cortex: enhanced theta synchrony in experts when watching a dance piece. Eur. J. Neurosci. 47, 433-445. https://doi.org/10.1111/ejn.13838

Poikonen, H., Toiviainen, P., Tervaniemi, M., 2016. Early auditory processing in musicians and dancers during a contemporary dance piece. Sci. Rep. 6. https://doi.org/10.1038/srep33056 
Porat, S., Goukasian, N., Hwang, K.S., Zanto, T., Do, T., Pierce, J., Joshi, S., Woo, E., Apostolova, L.G., 2016. Dance experience and associations with cortical gray matter thickness in the aging population. Dement. Geriatr. Cogn. Disord. EXTRA 6, 508517. https://doi.org/10.1159/000449130

Qi, M., Zhu, Y., Zhang, L., Wu, T., Wang, J., 2019. The effect of aerobic dance intervention on brain spontaneous activity in older adults with mild cognitive impairment: a resting-state functional MRI study. Exp. Ther. Med. 17, 715-722. https://doi.org/10.3892/etm.2018.7006

Ramanoël, S., Hoyau, E., Kauffmann, L., Renard, F., Pichat, C., Boudiaf, N., Krainik, A., Jaillard, A., Baciu, M., 2018. Gray matter volume and cognitive performance during normal aging. a voxel-based morphometry study. Front. Aging Neurosci. 10. https://doi.org/10.3389/fnagi.2018.00235

Reason, M., Jola, C., Kay, R., Reynolds, D., Kauppi, J.-P., Grobras, M.-H., Tohka, J., Pollick, F.E., 2016. Spectators' aesthetic experience of sound and movement in dance performance: a transdisciplinary investigation. Psychol. Aesthet. Creat. Arts 10, 4255. https://doi.org/10.1037/a0040032

Rehfeld, K., Hökelmann, A., Lüders, A., Kaufmann, J., Mueller, N., 2015. The effects of sixmonth exercise programs on structural changes in gray and white matter volume and balance abilities in senior citizens: the case for dance training. Front. Hum. Neurosci. 9. https://doi.org/10.3389/conf.fnhum.2015.217.00378

Rehfeld, K., Lüders, A., Hökelmann, A., Lessmann, V., Kaufmann, J., Brigadski, T., Müller, P., Müller, N.G., 2018. Dance training is superior to repetitive physical exercise in inducing brain plasticity in the elderly. PLOS ONE 13, e0196636. https://doi.org/10.1371/journal.pone.0196636

Rehfeld, K., Müller, P., Aye, N., Schmicker, M., Dordevic, M., Kaufmann, J., Hökelmann, A., Müller, N.G., 2017. Dancing or fitness sport? The effects of two training programs on hippocampal plasticity and balance abilities in healthy seniors. Front. Hum. Neurosci. 11. https://doi.org/10.3389/fnhum.2017.00305

Rizzolatti, G., Sinigaglia, C., 2010. The functional role of the parieto-frontal mirror circuit: interpretations and misinterpretations. Nat. Rev. Neurosci. 11, 264-274. https://doi.org/10.1038/nrn2805

Sacco, K., Cauda, F., Cerliani, L., Mate, D., Duca, S., Geminiani, G.C., 2006. Motor imagery of walking following training in locomotor attention. the effect of 'the tango lesson.' NeuroImage 32, 1441-1449. https://doi.org/10.1016/j.neuroimage.2006.05.018

Schmithorst, V.J., Wilke, M., Dardzinski, B.J., Holland, S.K., 2005. Cognitive functions correlate with white matter architecture in a normal pediatric population: A diffusion tensor MRI study. Hum. Brain Mapp. 26, 139-147. https://doi.org/10.1002/hbm.20149

Schubotz, R.I., Friederici, A.D., Yves von Cramon, D., 2000. Time perception and motor timing: a common cortical and subcortical basis revealed by fMRI. NeuroImage 11, 1-12. https://doi.org/10.1006/nimg.1999.0514

Schubotz, R.I., von Cramon, D.Y., 2001. Interval and ordinal properties of sequences are associated with distinct premotor areas. Cereb. Cortex 11, 210-222. https://doi.org/10.1093/cercor/11.3.210

Sejnoha Minsterova, A., Klobusiakova, P., Kropacova, S., Novakova, L., Brabenec, L., Balazova, Z., Grmela, R., Skotakova, A., Svobodova, L., Rektorova, I., 2020. Multishell diffusion MRI reflects improved physical fitness induced by dance intervention. Neural Plast. 2020. https://doi.org/10.1155/2020/8836925 
Senn, O., Kilchenmann, L., Bechtold, T., Hoesl, F., 2018. Groove in drum patterns as a function of both rhythmic properties and listeners' attitudes. PLOS ONE 13, e0199604. https://doi.org/10.1371/journal.pone.0199604

Shanahan, J., Morris, M.E., Bhriain, O.N., Saunders, J., Clifford, A.M., 2015. Dance for people with Parkinson disease: what is the evidence telling us? Arch. Phys. Med. Rehabil. 96, 141-153. https://doi.org/10.1016/j.apmr.2014.08.017

Spencer, P., 1985. Society and the Dance: The Social Anthropology of Process and Performance. Cambridge University Press, Cambridge.

Stark, E.A., Vuust, P., Kringelbach, M.L., 2018. Music, dance, and other art forms: new insights into the links between hedonia (pleasure) and eudaimonia (well-being). Prog. Brain Res. 237, 129-152. https://doi.org/10.1016/bs.pbr.2018.03.019

Stupacher, J., Hove, M.J., Janata, P., 2016. Audio features underlying perceived groove and sensorimotor synchronization in music. Music Percept. Interdiscip. J. 33, 571-589. https://doi.org/10.1525/mp.2016.33.5.571

Stupacher, J., Hove, M.J., Novembre, G., Schütz-Bosbach, S., Keller, P.E., 2013. Musical groove modulates motor cortex excitability: a TMS investigation. Brain Cogn. 82, 127-136. https://doi.org/10.1016/j.bandc.2013.03.003

Stupacher, J., Wood, G., Witte, M., 2018. Infectious Grooves: High-Groove Music Drives Auditory-Motor Interactions, in: Harrison, P.M.C. (Ed.), 2018 Proceedings of the 10th International Conference of Students of Systematic Musicology (SysMus17) (Print version: March 2018). Presented at the 10th International Conference of Students of Systematic Musicology (SysMus17), London, UK, September 13-15, 2017, p. 96: Zenodo. 10.5281/zenodo.1211651

Sumanapala, D.K., Walbrin, J., Kirsch, L.P., Cross, E.S., 2018. Neurodevelopmental perspectives on dance learning: insights from early adolescence and young adulthood, in: Christensen, J.F., Gomila, A. (Eds.), The Arts and The Brain: Psychology and Physiology Beyond Pleasure. Academic Press, Cambridge, MA, pp. 243-277. https://doi.org/10.1016/bs.pbr.2018.03.010

Tachibana, A., Noah, J.A., Bronner, S., Ono, Y., Onozuka, M., 2011. Parietal and temporal activity during a multimodal dance video game: an fNIRS study. Neurosci. Lett. https://doi.org/10.1016/j.neulet.2011.08.023

Tarr, B., Launay, J., Cohen, E., Dunbar, R., 2015. Synchrony and exertion during dance independently raise pain threshold and encourage social bonding. Biol. Lett. 11, 20150767. https://doi.org/10.1098/rsbl.2015.0767

Tarr, B., Launay, J., Dunbar, R.I.M., 2016. Silent disco: dancing in synchrony leads to elevated pain thresholds and social closeness. Evol. Hum. Behav. 37, 343-349. https://doi.org/10.1016/j.evolhumbehav.2016.02.004

Teki, S., Grube, M., Kumar, S., Griffiths, T.D., 2011. Distinct neural substrates of durationbased and beat-based auditory timing. J. Neurosci. 31, 3805-3812. https://doi.org/10.1523/JNEUROSCI.5561-10.2011

Thaut, M.H., Trimarchi, P.D., Parsons, L.M., 2014. Human brain basis of musical rhythm perception: common and distinct neural substrates for meter, tempo, and pattern. Brain Sci. 4, 428-452. https://doi.org/10.3390/brainsci4020428

Trost, W., Frühholz, S., Schön, D., Labbé, C., Pichon, S., Grandjean, D., Vuilleumier, P., 2014. Getting the beat: Entrainment of brain activity by musical rhythm and pleasantness. NeuroImage 103, 55-64. https://doi.org/10.1016/j.neuroimage.2014.09.009

Ushiyama, J., Takahashi, Y., Ushiba, J., 2010. Muscle dependency of corticomuscular coherence in upper and lower limb muscles and training-related alterations in ballet 
dancers and weightlifters. J. Appl. Physiol. https://doi.org/10.1152/japplphysiol.00869.2009

Ventouras, E.-C., Margariti, A., Chondraki, P., Kalatzis, I., Economou, N.-T., Tsekou, H., Paparrigopoulos, T., Ktonas, P., 2015. EEG-based investigation of brain connectivity changes in psychotic patients undergoing the primitive expression form of dance therapy: a methodological pilot study. Cogn. Neurodyn. 9, 231-248. https://doi.org/10.1007/s11571-014-9319-8

Vuust, P., Frith, C.D., 2008. Anticipation is the key to understanding music and the effects of music on emotion. Behav. Brain Sci. 31, 599-600. https://doi.org/10.1017/S0140525X08005542

Vuust, P., Heggli, O.A., Friston, K.J., Kringelbach, M.L., 2021. Music in the brain. Submitted.

Vuust, P., Ostergaard, L., Pallesen, K.J., Bailey, C., Roepstorff, A., 2009. Predictive coding of music - brain responses to rhythmic incongruity. Cortex, Special Issue on "The Rhythmic Brain" 45, 80-92. https://doi.org/10.1016/j.cortex.2008.05.014

Vuust, P., Roepstorff, A., Wallentin, M., Mouridsen, K., Østergaard, L., 2006. It don't mean a thing... Keeping the rhythm during polyrhythmic tension, activates language areas (BA47). NeuroImage 31, 832-841. https://doi.org/10.1016/j.neuroimage.2005.12.037

Vuust, P., Witek, M.A.G., 2014. Rhythmic complexity and predictive coding: a novel approach to modeling rhythm and meter perception in music. Front. Psychol. 5. https://doi.org/10.3389/fpsyg.2014.01111

Wind, J., Horst, F., Rizzi, N., John, A., Schöllhorn, W.I., 2020. Electrical brain activity and its functional connectivity in the physical execution of modern jazz dance. Front. Psychol. 11. https://doi.org/10.3389/fpsyg.2020.586076

Witek, M.A.G., 2017. Filling in: syncopation, pleasure and distributed embodiment in groove. Music Anal. 36, 138-160. https://doi.org/10.1111/musa.12082

Witek, M.A.G., Clarke, E.F., Wallentin, M., Kringelbach, M.L., Vuust, P., 2014. Syncopation, body-movement and pleasure in groove music. PLOS ONE 9, e94446. https://doi.org/10.1371/journal.pone.0094446

Zilidou, V.I., Frantzidis, C.A., Romanopoulou, E.D., Paraskevopoulos, E., Douka, S., Bamidis, P.D., 2018. Functional re-organization of cortical networks of senior citizens after a 24-week traditional dance program. Front. Aging Neurosci. 10. https://doi.org/10.3389/fnagi.2018.00422 